\title{
Towards Mitigating Broadcast Storm in Vehicular Content Centric Networks for Big Data Analytics
}

\author{
Abdul Wahid ${ }^{\mathrm{a}}$, Munam Ali Shah ${ }^{\mathrm{a}}$, Faisal Fayyaz Qureshi ${ }^{\mathrm{b}},{\text { Hafsa } \text { Maryam }^{\mathrm{a}} \text {, Rahat Iqbal }}^{\mathrm{b}}$, Victor \\ Chang ${ }^{\mathrm{c}}$ \\ ${ }^{a}$ Department of Computer Science COMSATS Institute of Information Technology, Islamabad, Pakistan \\ ${ }^{b}$ Coventry Univesity, UK and Smart Innovation Techno Center, UK \\ ${ }^{c} I B S S, X i$ 'an Jiaotong-Liverpool Univesity, Suzhou, China
}

\begin{abstract}
The focus of the research for big data analytics for sustainability (BDAS) is Vehicular Ad-hoc Networks (VANETs), since it has become the most popular technology for the researchers, academia and the organizations. However, in VANETs most part of the time a well-defined path from a consumer to the provider doesn't exists due to the intermittent connectivity and mobility. Hence, Vehicular Content Centric Networks (VCCN) has been proposed and has emerged as a future Internet technology. The focus of the VCCN is to retrieve and distribute the content faster than the existing Internet architecture in vehicular environment. In VCCN, consumer broadcasts the interest packet to the provider including the name of the required content. If the provider is not available at one hop neighbor, then the interest packet is sent to the forwarder vehicle, otherwise, provider generates the data packet and sends back to the consumer. Forwarder vehicle receives this interest packet from the consumer and searches in its cache called Content Storage (CS). If the content is not available in its storage the forwarder vehicle rebroadcasts the interest packet within its vicinity and forward the interest to the provider. The provider generates the data packet including the content and sends back to the consumer through selected forwarder vehicle. The VCCN communication paradigm faces several challenges i.e., mobility, interest broadcasting storm, routing, interest/data packet forwarding and forwarder selection. Various routing protocols have already been proposed and implemented to resolve existing problems. However, in our research due to the high mobility and the dynamic network topology in vehicular environment, there is no assurance that the back path is still available followed by the interest message which causes disconnected link problem. Hence, the retrieval of the required data might be effected. Thus, the aim of this paper is to propose a forwarding protocol to mitigate the interest broadcasting storm and the disconnected link problems, keeping in the view of intermittent connectivity and high mobility that will improve the data delivery probability, packet loss ratio, Interest Satisfaction Ratio (ISR) and average End-to-End delay in a highway and an urban scenario of a vehicular environment. Results and demonstrations show that the proposed protocol can meet requirements for BDAS.
\end{abstract}

Keywords: Forwarder; Routing; Mobility; Big Data Analytics for Sustainability (BDAS); Vehicular Content Centric Networks (VCCN).

Email addresses: abdulwahid@comsats.edu.pk (Abdul Wahid), mshah@comsats.edu.pk (Munam Ali Shah), 


\section{Introduction}

This paper addresses big data analytic for sustainability (BDAS) as follows. Nowadays, the Internet plays a very important role in various aspects of academics, industries, government and users lives. Despite the commercial success of the existing Internet, the usage of Internet is growing day by day and is becoming more content-centric in order to fulfil the new emerging needs i.e., mobility, trust, content distribution, security and privacy from the existing communication paradigm. We require a new architecture to enhance the existing infrastructure of the Internet because the current host-to-host communication paradigm never aimed to deal such type of requirements. The existing Internet could only establish connectivity between two static endpoints (hosts) which are determined by its IP addresses. Hence, host-to-host communication model is a location dependent network model. Moreover, host-to-host communication model needs to specify the address of the device in every request. Also, it must identify the address of the server from which the content can be accessed. Current Internet is based on a location-dependent model. However, locationdependent communication model does not support mobility and privacy because these features are not built-in fundamentally and are available in the form of different 'patches'which may fail at any time. Moreover, patches enhance the complexity of the overall paradigm and sometimes use as a temporary solution as shown in Fig. 1 [1][2].

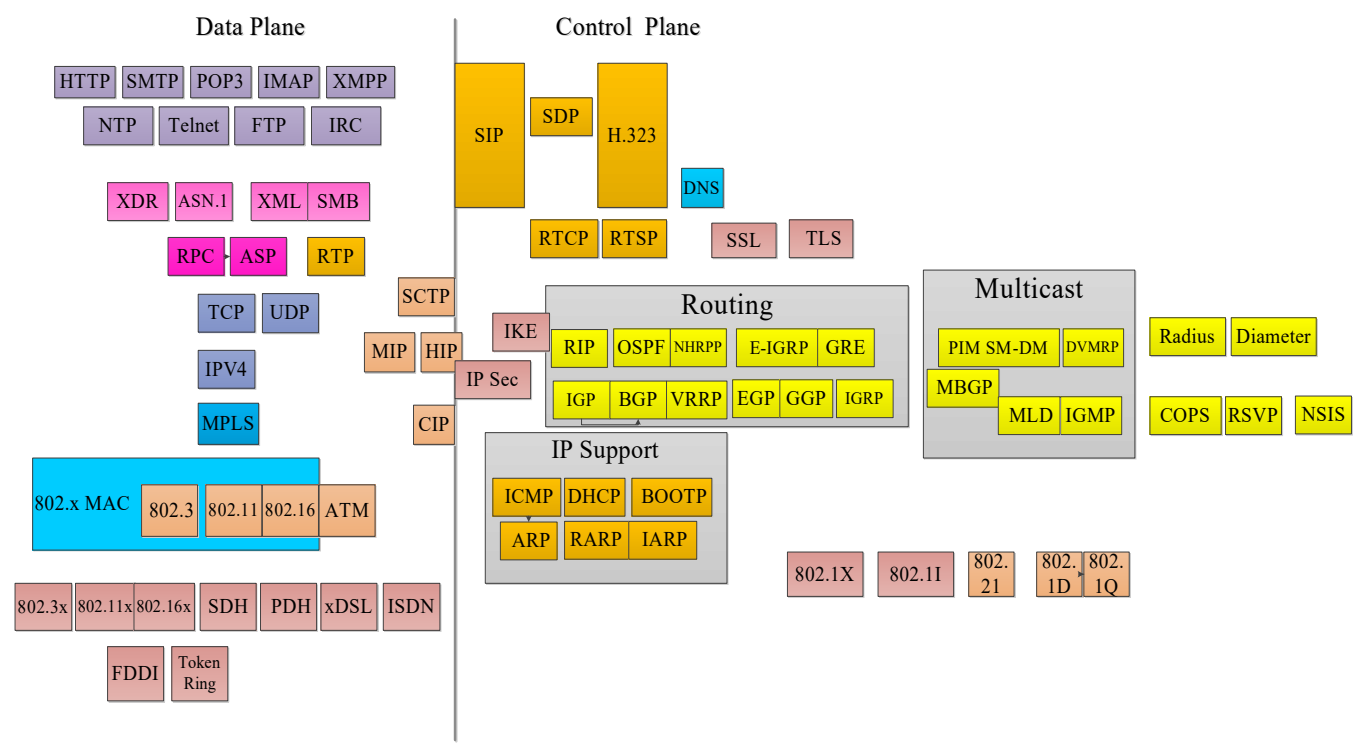

Figure 1: Internet architecture in the form of patches based on [1][2]

It is challenging to design, develop and incorporate new requirements into the existing Internet architecture [3][4]. The research community has been identifying the limitations of the existing Internet architecture. They highlight the requirements of the future Internet architecture and

faisalf2001@yahoo.com (Faisal Fayyaz Qureshi), hafsa.maryam09@gmail.com (Hafsa Maryam), r.iqbal@coventry.ac.uk (Rahat Iqbal), victorchang.research@gmail.com (Victor Chang) 
propose a new emerging paradigm, i.e., Information Centric Network (ICN) which addresses the existing Internet communication paradigm challenges [5][6]. The ICN has attracted the researchers because of the capability to easily overcome the challenges in the host-to-host communication networks. Basically, the ICN shifts from the host-centric paradigm to content-centric paradigm in which the retrieval of the content is the main motivation. The basic aim of the ICN is to reflect the future and the present needs better than the existing host-centric paradigm and timely and efficient delivery of the content to the individuals [7][8]. ICN is based on the location-independent model in which a user can only request the content by its name. ICN provides many potential services i.e., security, naming, content delivery, mobility and privacy which are inherently built into the communication network system [9][5][6]. The initiatives of the ICN paradigm focused on developing the content-centric paradigm [3][4]. Different initiatives of the ICN projects have been classified into four generations as shown in Fig. 2.

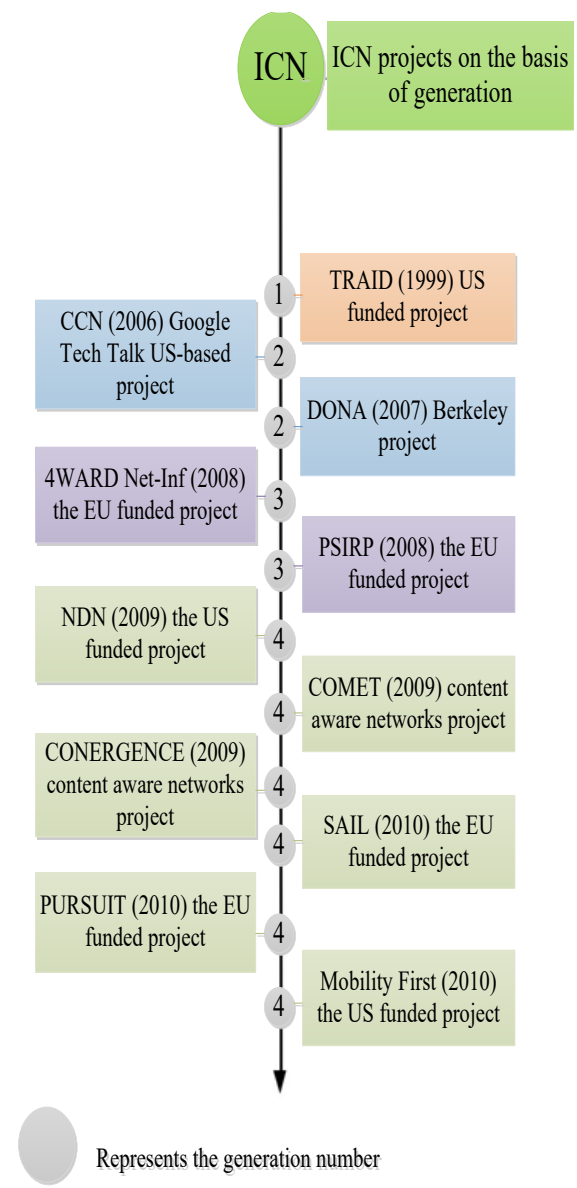

Figure 2: ICN projects on the basis of the time-line

The first ICN project is TRAID [10] which introduced the content-based routing on the top of the IP layer. In second generation, the initiatives of ICN introduced the DONA [11], motivated by TRAIT. DONA extends the content-based routing, but naming scheme was replaced with flat 
names and the hierarchal URL. In third generation, PSIRP [12] and Net-Inf [13] were developed for the inter-domain and intra-domain levels. In fourth generation, PURSUIT, CONNECT, SAIL [14], CONVERGENCE, NDN [15] are classified and developed based on the continuous efforts which were carried out in [16][4]. The Content Centric Networking (CCN) [2][3] is also included in the second generation of ICN which has been the main objective to shift from location-centric to content-centric networks. This transition of a host-centric approach to a content-centric approach is represented in Fig. 3.

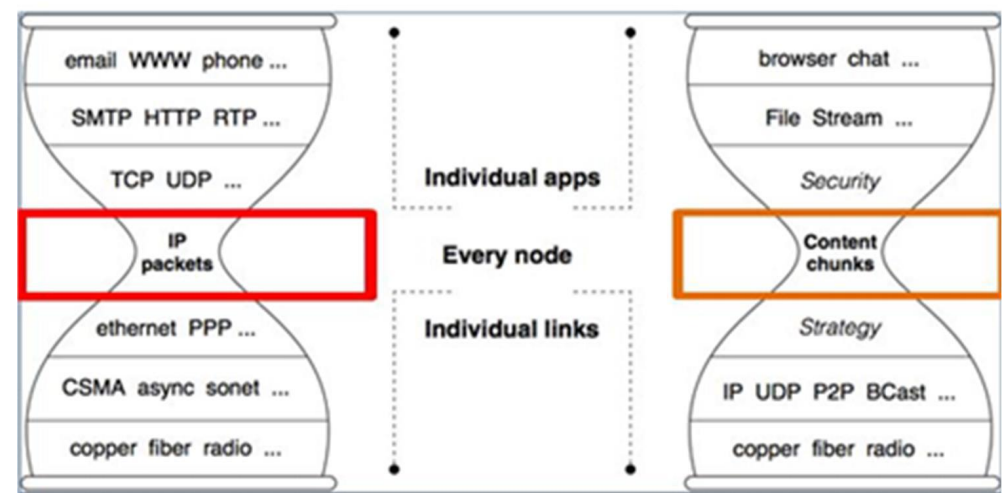

Figure 3: The change of the IP layer with the content chunks in a CCN taken from [3]

The Internet distributes the information to a large number of individuals usually from a single source. This results in similar information being requested thousands of times from the same source which ultimately looses lots of useful resources. The CCN addresses this issue efficiently as each object in a CCN has its own unique identifier and each object has the equal right to request any content by its own unique name of the layer of the network. This gives the direct access of the information to the requester and the contents are delivered in an effective way.

The recent advanced developments in the vehicular industry has given rise to Vehicular Ad-hoc Networks (VANETs) [17][18]. The applications of VANETs are majorly classified as safety and non-safety applications. Safety vehicular applications consist of lane changing, before-crash sensing, stop sign movement, emergency and traffic signal violation. Safety messages are derived from these applications and they need direct communication with the infrastructure [19]. These safety-based messages give real time information. The non-safety vehicular applications are consisted of the commercial, comfort, gas stations, hotels, electronic toll collection, weather information and parking lot etc. applications [20]. In VANETs, intelligent vehicles contain a lot of sensors and applicable hardware to take decision wisely, particularly for the benefits of the drivers. This is the reason, the VANETs are also called an Intelligent Transportation System (ITS) [21]. The objectives of an ITS is to enhance the urban and highway awareness, entertainment in transportation and to try to improve the safety of the user by adapting new technologies for communication and take different types of information. There are different types of communications in a VANET namely vehicle-tovehicle communication (V2V); vehicle-to-infrastructure communication (V2I) and communication with the Road Side Units (RSUs) as shown in Figure 4. VANETs paradigm faces many challenges, i.e., high mobility, intermittent connectivity between vehicles due to changing their velocity and direction constantly. It is very difficult to judge the position of the host providing services [22]. 
Moreover, in a vehicular environment, a well-defined path from a source to the provider does not exist most of the time. According to the above-mentioned issues, a CCN is the best paradigm to share resources in VANETs paradigm. This is the main reason, the research community has adapted the CCN paradigm in VANETs named as Vehicular Content Centric Networks (VCCN).

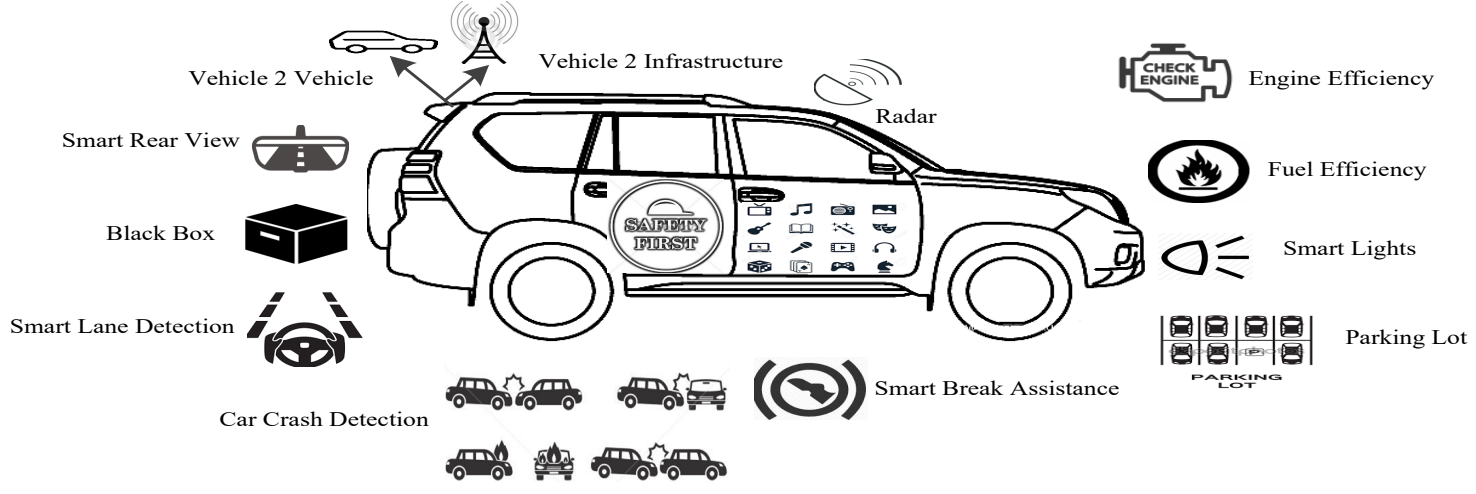

Figure 4: Intelligent vehicle contains various components

VCCN has emerged as a future technology for the researchers, organizations, industries and the academia [23]. Furthermore, content access and distribution becomes easier and faster than the existing host-to-host communication paradigm [24][25]. Despite, numerous benefits of a VCCNs, there are different challenges faced by the VCCN [26]. We represent these challenges in Fig. 5 and briefly discuss them below.

1. Mobility: In a VCCN architecture, the communication between two vehicles can be done on the basis of the naming scheme. However, more demanding techniques needs to be proposed and implemented to overcome the high mobility, intermittent connectivity and the dynamic network topology.

2. Interest Broadcast Storm: In VCCNs, when consumer sends an interest packet to the provider, if provider is available in one hop neighbor, the provider sends the data packet immediately to the consumer otherwise, interest message is disseminated into the whole network to find a provider. This dissemination causes interest broadcast storm and congestion in a VCCN topology and results in the instability of the network. The rate of the transmission of the interest messages needs to be controlled according to the available resources provided by the network.

3. Routing: Content based routing is one of the hottest and debatable topic in VCCNs which aims to achieve effective Quality of Service (QoS) in a highly dynamic network topology. The selection of the forwarder, sending a response and the receipt of a reply between consumer 


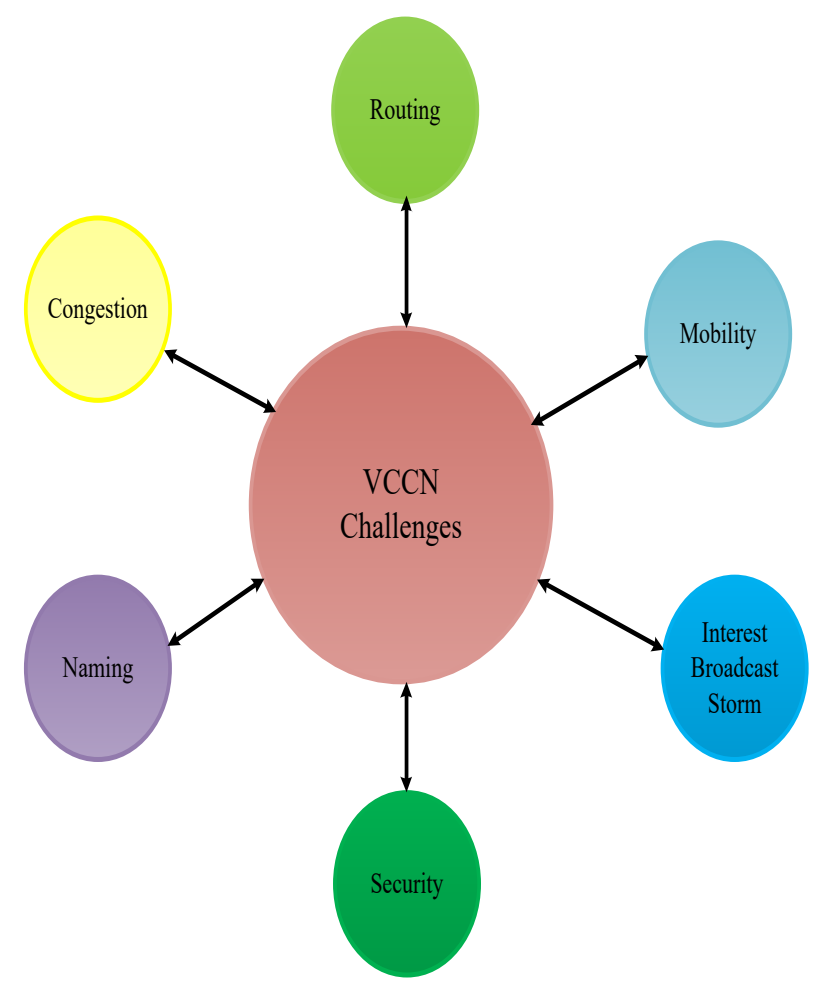

Figure 5: Different challenges of the VCCN

and provider is the major responsibility of any routing strategy. The VCCNs need an effective content routing scheme to fulfill the request of the desire content efficiently and effectively.

4. Congestion: In VCCNs, if the forwarding of an interest message can be completed easily without selecting the best forwarder within the vicinity of each vehicle, then multiple interest packets may be pipelined in the form of a queue which causes congestion in VCCNs. The best forwarder needs to be selected within the available transmission range of each vehicle in order to avoid congestion.

5. Naming: In CCNs, there are different naming schemes which can be used for VCCNs. These naming schemes are categorized as flat, hierarchical, human-readable, hash-based and hybrid naming schemes. Which scheme is more suitable for VCCNs is still an open issue.

Many routing protocols have been proposed to overcome the challenges such as mobility, dynamic network topology, forwarding selection and the interest/data packet forwarding [26], however, due to the dynamic network topology and the mobility, there is no assurance that the back track is still available followed by the interest message. Hence, there is a lot of risk that the access of a particular content might be affected [27] [28][29].

The motivation of this research is to develop a routing protocol that is able to overcome the challenges regarding routing, interest/packet forwarding and forwarder selection interest broadcast storm and disconnected link problems in VCCNs. The goals of this research are as follows.

- To develop a routing protocol that is capable to eliminate the broadcast storm and discon- 
nected link problems in VCCN.

- To develop a routing protocol that ensures the maximum content packet successfully receives at the consumer.

- To enhance a routing protocol which makes decision about choosing the best forwarder and provides the stability of the network.

- To evaluate the performance of the proposed routing protocol and compare with another existing routing protocol in VCCNs such as Robust Forwarder Selection (RUFS) [27] for the parameters such as Packet Delivery Ratio (PDR), Packet Loss Ratio (PLR), Interest Satisfaction Ratio (ISR), and Average End-to-End Delay.

\section{Literature Review}

Big data analytics is the process of analyzing huge amount of data which can be used for different purposes. Some of the objectives of big data analytics could be to reveal hidden patterns, to know the unspecified relations and to forecast business trends etc. The sustainability aspect of the big data focuses on the future of society, environment and commercialism to improve humans life standards. This section describes a list of related literature for big data analytics for sustainability (BDAS), with particular focus for Vehicular Content Centric Networks (VCCN). Fig. 6 shows the relationship between BDAS and VCCN. It can be observed that several important decisions can be made in the VCCN environment with the help of BDAS which ultimately brings improvements in the lifestyle.This section describes a list of related literature for big data analytics for sustainability (BDAS), with particular focus for VCCN.

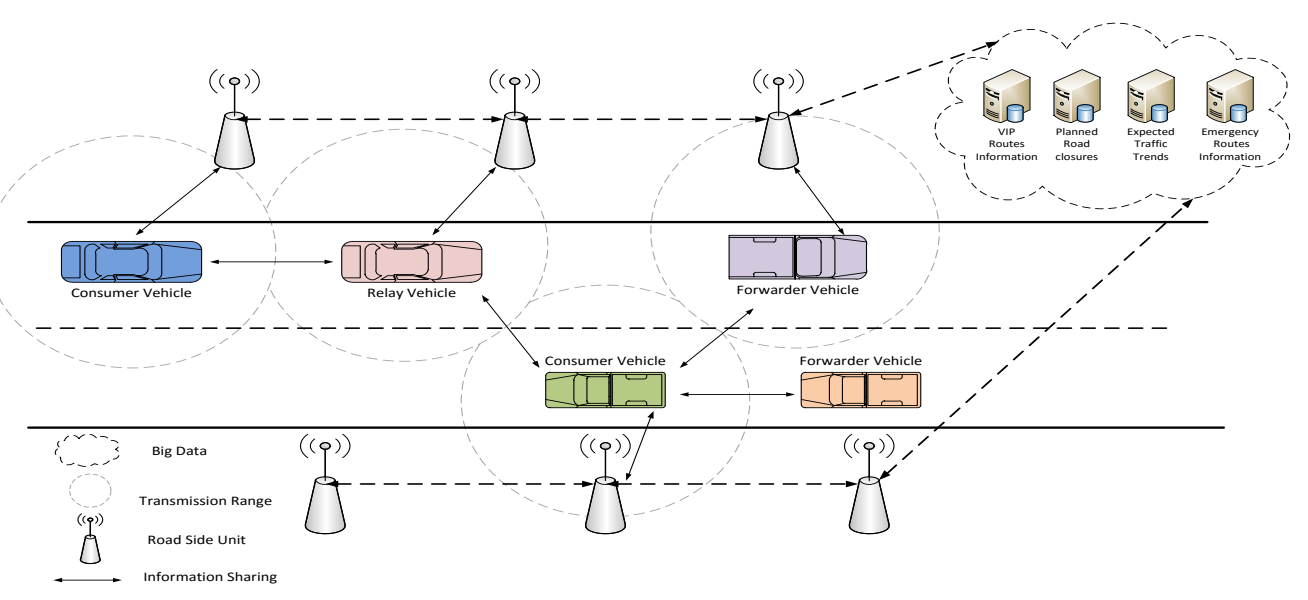

Figure 6: Relationship between BDAS and VCCN

Fabrcio et al. [30] proposed a new content-centric model for the vehicular environments called Interest Centric Mobile Ad-hoc Networks for Vehicular Networks (RadNet-VE). In this proposed model, the author introduced a unique data structure named active prefix. Each node contains the active prefix data structure in the network layer. Active prefix consists of an application interest 
and a node prefix. Application interest is used for the formation of a group and a name searching, whereas, node prefix is used for the identification of the node, message forwarding and addressing. Basically, the author extended the header of the interest message and introduced the scheme for registering the interest message. The scheme forwards the message on the basis of the direction of the propagation of the interest message and the negative position of the source. This model achieves the less delay and high delivery ratio. The limitation of the scheme is each node does not store any data and no in-network cache scheme is performed at individual nodes. Another limitation is the limited scope of the interest message communication and the membership service is implemented in a distributed manner.

Farhan et al. [31] proposed a new forwarding scheme named as Push Vehicular Named Data Networking (P-VNDN). In case of any accident, the vehicle broadcasts an interest message as an emergency content to the near RSU. Upon receiving the emergency message. the RSU compares the header of the message with its own contents available in its cache called Content Storage (CS). In the case of a match, the RSU processes this content otherwise, forwards this message to the other RSUs that is not available in the range of a requester vehicle. In case of no RSU, intermediate vehicle carries forward this emergency message until an RSU is reached in the range of a forwarder vehicle. The P-VNDN uses a proactive approach to disseminate the data in a vehicular environment. This forwarding scheme allows the vehicles to disseminate an emergency message up to one-hop neighbor. P-VNDN achieves the less delay than the Pull-VNDN. The limitation of this scheme is no mobility mechanism is defined at an intermediate node, especially at the reverse path (i.e., from a provider to the consumer) in case, when forwarder vehicle moves away from the consumer.

Saxena et al. [32] implemented an IP-based forwarding scheme using Named Data Networking (NDN) in a vehicular environment. Author's goal is to analyze the performance of the NDN forwarding scheme to access and to disseminate of the content. VNDN model achieves the usability of the NDN in vehicular environment. In this model, the authors shows the performance comparison among the NDN-based algorithms, i.e., Epidemic, Adaptive and Spray \& Wait.

Khaleel et al. [33] proposed the receiver-based forwarding scheme in which they addressed the challenges of making the unnecessary multiple paths especially when forwarder vehicles are not in the transmission range of the other vehicles in a VANET environment. Authors selected the forwarder vehicles based on a selection metrics i.e., Forwarding Zone and Signal Interference Noise Ratio (SNIR). When a receiving node receives the packet, the node matches the SNIR value with the already defined threshold value and compares the forwarding angle with the angle that is already defined in the packet. If the value of the SNIR is greater and value of angle is less than already defined value in the packet, this node is called as a best forwarder, otherwise, the forwarder drops the packet and delete the timer. Forwarder vehicle sets its own timer and rebroadcasts the packet within its vicinity. The forwarding scheme selects the neighbor node as a best forwarder who is nearest to the destination. In this scheme, the authors prevent the formation of unnecessary multiple paths successfully and also reduce the congestion in the whole network by using the shortest path in the network. The limitation of this forwarding scheme is lot of retransmissions of the packets which create the more end-to-end delay in the network.

Lee et al. [34] proposed the interest forwarding scheme named parallel multi-path interest forwarding scheme to achieve the high transmission rate in a CCN. In this scheme, a requester requests the interest message to the client based on Round-Trip Time (RTT) value. After receiving the message, the client parallelly processes the chunks of the requested content from different wireless interfaces. In this way, the client achieves the higher transmission rate than the other 
clients. In this scheme, each server has its own cache repository for cache replacement and a decision policy. This scheme applies Leave Copy Every Where Policy for cache decisions and for the replacement it applies the Leave Recently Used Policy. The forwarding scheme compares the three different algorithms among each other to achieve less delay. The limitation of this scheme is disconnected link problems due to the reason that client (mobile node) moves away from the source (i.e., a server).

Yan et al. [35] proposed a model of a Vehicular Information Network (VIN) based on the basic principal of NDNs [36][37][38]. The VIN extends the concept of an NDN and apply on the vehicular networks for the fast dissemination of vehicular information [15]. The proposed model has a hierarchical content naming scheme, distributed mobility management scheme and location-based forwarding which is dependent on the name of the content for the VIN. The authors have set the time on the router to assemble different data packets. The value of the timer is inherited from the upper-level to the lower-level aggregator in the interest packet. Authors have also analyzed some new challenging features such as low communication efficiency and high information management overhead within the VIN. Yan et al. research aims to develop an architecture which is more suitable for distributing, allocating and accessing contents efficiently and effectively from a large area with more number of vehicles. The paper lags in providing any detailed description of data and interest packet forwarding strategies.

Tian et al. implemented a protocol named Traffic Adaptive Data Dissemination (TrAD) [39]. This protocol focuses on the V2V communication instead of V2I communication for both urban and highway scenarios. This scheme overcomes the issue of broadcasting storm in dense environment. This protocol operations in two phases: textit(i) a time slotted broadcasting mechanism is used to overcome the issue of broadcasting storm. The authors claim that this phase enhances the transmission reliability; ii) the best forwarder is elected to further limit the duplicate transmission. The proposed protocol results in lesser delay and higher PDR. No mobility mechanism is defined when a stationery forwarder vehicle changes its position and moves away from the sender/coordinator.

Lin et al. [40] proposed a forwarding scheme named Reliable Forwarding Strategy (RFS). The main objective of this scheme is to provide a reliable path from source to destination and vice versa. The scheme ensures that all the forwarder nodes in the path of the interest messages remain active whenever the data packet is sent back to the consumer followed by the path of the interest message. Furthermore, the RFS reduces the overhead of the entire network by using the beacon message to exchange the information between the intermediate nodes. In this scheme, best forwarder selection is done in such a way that each node calculates its destination from the sender. If the distance is greater, it becomes the best forwarder and then the forwarder node sets a waiting timer. When the timer reaches the specified value the best forwarder node broadcasts the message in its vicinity. RFS provides better results using the concept of the waiting timer however, this scheme does not provide details of the reverse path when the provider sends the data packet to the consumer resulting in the transmission delay in the network.

Wang et al. [41] proposed a V2V communication model using an NDN architecture. This model addresses different limitations and challenges faced by the VNDN. In this scheme, authors describe the $\mathrm{V} 2 \mathrm{~V}$ applications in different categories by using NDN communication paradigm. The scheme does not focus which traffic application category would be used in this model.

Wang et al. [42] addresses the multi-part communication issue by applying NDN concept to V2V communication to minimize collision. Authors deployed an application to provide successful data propagation, named as Rapid Traffic Information Dissemination by using name-data concept. 
Author's motivation is to disseminate the packet farther away efficiently, with minimum overhead from the origin. Furthermore, the authors consider a situation when a nearby vehicle rebroadcast the packet. Their simulation results show that the interest forwarding and the retrieval of the content based on NDN is more rapid, reliable and scalable than the host-to-host based V2V communication.

Ko et al. [43] proposed a position-based forwarding scheme. In this scheme, forwarding is decided on the basis of the location of the consumer, location of the provider and the area of the provider. The consumer selects the forwarding area between a consumer and the provider areas. All nodes within the selected area must forward the message while the other nodes that are outside of this selected area discard the message. This scheme achieves the high delivery ratio and low communication overhead.

S.Hassan et al. [44] proposed a 'Traffic Violation Ticketing (TVT)'system in order to cope an anonymous vehicle who violates the traffic rules. In order to become this system more efficient authors have considered that each vehicle is equipped with the automatic ticket generating device. The main objective of this scheme is to catch the vehicle that violate the traffic rules i.e, parking lot and all the information related to violence received from the neighbouring vehicles. In this scheme, VCCN communication paradigm apply on Traffic Violation Ticketing system in such a way traffic violating vehicle broadcasts an interest packet periodically and all the entries related to traffic violation maintain in the Pending Ticket Entry (PTE) table. In order to exchange the PTE table traffic violated vehicle send a ticket to the offender. When offender vehicle reaches with in the vicinity of the RSU then pay the charges against the violation ticket. The limitation of this scheme is there is no simulation scenario is performed in order to generate some realistic result. TVT system was only applied on the intermediate neighbour node but in real simulation environment, there is a need to implement the interest flooding mechanism. TVT system ignores the delay and as well as manual operations error caused by the human [45].

G. Denge et al. [46] proposed the hybrid forwarder scheme in VANET by using NDN architecture, named Hybrid Vehicular Named Data Networking (HVNDN).HVNDN takes the operations of NDN architecture and geographic information in VANET.HVNDN categorize the transmitted information in to two types in vehicular environment[47]. First type is location in dependent and the other type is location dependent forwarder mechanism. Forwarding strategy introduced the new mechanism acknowledgement and retransmission for the interest and data packet forwarding, the reason behind to delivered the packet successfully and reliably. Forwarding scheme achieves the less end-to-end delay, hop-count and high PDR as compared to the other existing protocols. The limitation of this scheme is authors did not consider any mobility mechanism at the forwarder node in such a way when forwarder vehicles move away from the consumer.

Amadeo et al. [48][49] proposed the forwarding scheme named Content-Centric Vehicular Network (CCVN) for vehicular environment. In this scheme [49], the authors categorized the interest packet into two parts. First, the basic interest packet and second, the advanced interest packet. In this scheme, basic interest packet is sent when a source wants to require a particular content. In advanced, the packet is to find the last content discovered destinations. Moreover, in this forwarding scheme authors introduced a Content Provider Table (CPT). The CPT replaces the Forwarding Information Based (FIB) table. The CPT stores the information that has already been determined and associated with the address at MAC layer. The forwarding scheme efficiently achieves the load balancing however, the modification in the $\mathrm{CCN}$ architecture can compromise the mobility of the vehicles. 
Garris et al. [50] implemented an interest forwarding location-based scheme named Navigo for the VNDN. Basically, authors have focused on the geographical information. They find sources and then forward the interest packet along the shortest and best path, considering the special characteristics of the vehicular environment. The main idea of this forwarding scheme is to find the geographical location surrounded by the vehicle looking for a producer and a RSU to get the data packet faster. Geographic location finds the area to identify the origin of the data packet. This scheme achieves better packet delivery but the limitation of this scheme is the assumption that all RSUs listen to the interest packet within its Wi-Fi transmission range. However, realistically the situation may be different from that because RSUs may not be in the range of the vehicles. In this case, the vehicles may not send or receive the packets.

Faisal at al. [51] implemented a reliable and energy efficient communication using cognitive radio technology. Authors have focused on reliable channel selection, however, no description has been provided about the selection technique in vehicular environment when the speed of a vehicle is changing frequently.

In this section, we discussed the forwarding protocols for big data analytics for sustainability (BDAS) with focus for VCCN, VANET, CCN and VNDN in detail. We have analyzed the research paper from 2010-2017. In Table 1, we provide a brief and concise review of different routing strategies. It could be observed that in the existing research, due to the mobility, there is no guarantee that the reverse path is still available followed by the interest packet. Hence, the retrieval of required contents is affected and cause disconnected link problem. Some of the research papers provide less delay but no mobility mechanism is defined at intermediate nodes, especially at the reverse path form provider to consumer. Some paper mitigated the interest broadcasting storm but don't provide disconnected link problem solution. So, there is a need to suggest a new protocol in which the interest broadcast storm issue is mitigated, the mobility mechanism is defined at intermediate nodes through metrics selection and which also addresses the disconnected link problem. 
Table 1: Literature Review of Different Forwarding Scheme.

\begin{tabular}{|c|c|c|c|c|}
\hline $\begin{array}{l}\text { Author } \\
\text { Name }\end{array}$ & $\begin{array}{l}\text { Proposed } \\
\text { Scheme Name }\end{array}$ & Proposed Scheme & Benefits & Limitations \\
\hline $\begin{array}{l}\text { Fabrcio } \\
\text { et al. } \\
(\mathbf{2 0 1 7})\end{array}$ & $\begin{array}{l}\text { Interest Centric[30] } \\
\text { Mobile Ad- } \\
\text { hoc Networks } \\
\text { for Vehicular } \\
\text { (RadNet-VE) }\end{array}$ & $\begin{array}{l}\text { RadNet-VE has a unique data structure } \\
\text { named Active prefix.Author's have ex- } \\
\text { tended the headers of the interest mes- } \\
\text { sage. }\end{array}$ & $\begin{array}{l}\text { Achieves Less delay and } \\
\text { high delivery ratio. }\end{array}$ & $\begin{array}{l}\text { Interest message mem- } \\
\text { bership service is imple- } \\
\text { mented in a distributed } \\
\text { manner. }\end{array}$ \\
\hline $\begin{array}{l}\text { Farhan } \\
\text { et al. } \\
(\mathbf{2 0 1 7 )}\end{array}$ & $\begin{array}{lr}\text { Push } & \text { vehic- } \\
\text { ular } & \text { Named } \\
\text { Data } & \text { Networks } \\
(\text { P-VNDN) [31] }\end{array}$ & $\begin{array}{l}\text { In proposed scheme named PVNDVN, } \\
\text { RSU compares the header of the mes- } \\
\text { sage with its own cache content upon re- } \\
\text { ception. RSU keeps this content if match, } \\
\text { otherwise forwards it to the other RSU. }\end{array}$ & $\begin{array}{l}\text { Achieves less delay than } \\
\text { the pull VNDN. }\end{array}$ & $\begin{array}{l}\text { Mobility of intermediate } \\
\text { nodes at the reverse path } \\
\text { has not been considered. }\end{array}$ \\
\hline $\begin{array}{l}\text { Khaleel } \\
\text { et al. } \\
(\mathbf{2 0 1 7})\end{array}$ & $\begin{array}{l}\text { Receiver based } \\
\text { Forwarding } \\
\text { Scheme [33] }\end{array}$ & $\begin{array}{l}\text { Consumer selects the neighbor node as a } \\
\text { forwarder who is nearest to the destina- } \\
\text { tion. }\end{array}$ & $\begin{array}{l}\text { Author's have mini- } \\
\text { mized the unnecessary } \\
\text { formation of multi- } \\
\text { ple paths towards the } \\
\text { destination. }\end{array}$ & $\begin{array}{l}\text { A lot of packet retrans- } \\
\text { mission creates the more } \\
\text { end-to-end delay in the } \\
\text { network. }\end{array}$ \\
\hline $\begin{array}{l}\text { Lee } \\
\text { et al. } \\
(\mathbf{2 0 1 7})\end{array}$ & $\begin{array}{l}\text { Parallel Multi- } \\
\text { path Interest } \\
\text { Forwarding } \\
\text { Scheme [34] }\end{array}$ & $\begin{array}{l}\text { In this scheme,client chooses the router } \\
\text { on the basis of RTT value to send interest } \\
\text { message. }\end{array}$ & Achieves less Delay. & $\begin{array}{l}\text { Causes disconnected link } \\
\text { problem. }\end{array}$ \\
\hline $\begin{array}{l}\text { Lin } \\
\text { et al. } \\
(\mathbf{2 0 1 7})\end{array}$ & $\begin{array}{lr}\text { Reliable } & \text { For- } \\
\text { warding } & \text { Strat- } \\
\text { egy } & \text { (RFS) } \\
{[40]} & \end{array}$ & $\begin{array}{l}\text { In RFS, consumer selects the neighbor } \\
\text { vehicle with larger distance as the best } \\
\text { forwarder. Forwarder vehicle broadcasts } \\
\text { the message with in its vicinity after a } \\
\text { pre-calculated time. }\end{array}$ & $\begin{array}{l}\text { Provide the reliable path } \\
\text { between source and des- } \\
\text { tination. }\end{array}$ & $\begin{array}{l}\text { No timer scheme is ap- } \\
\text { plied at reverse path } \\
\text { which causes transmis- } \\
\text { sion delay in the net- } \\
\text { work. }\end{array}$ \\
\hline $\begin{array}{l}\text { Hassan } \\
\text { et al. } \\
(\mathbf{2 0 1 5 )}\end{array}$ & $\begin{array}{l}\text { Robust For- } \\
\text { warder Selection } \\
\text { (RUFS)[27] }\end{array}$ & $\begin{array}{l}\text { Each vehicle maintains two types of } \\
\text { list.First RSL, which maintains a list of } \\
\text { interest satisfied by a vehicle. Other is } \\
\text { NSL, which maintains a list of interest } \\
\text { satisfied by vehicle's neighbors. }\end{array}$ & $\begin{array}{l}\text { Overcomes conges- } \\
\text { tion,achieves less delay } \\
\text { and mitigate interest } \\
\text { broadcast storm. }\end{array}$ & $\begin{array}{l}\text { This scheme causes dis- } \\
\text { connected link problem } \\
\text { and content retrieval } \\
\text { failure. }\end{array}$ \\
\hline $\begin{array}{l}\text { Hassan } \\
\text { et al. } \\
(\mathbf{2 0 1 6 )}\end{array}$ & $\begin{array}{l}\text { Controlled } \\
\text { Data Packets } \\
\text { Propagation } \\
\text { in Vehicu- } \\
\text { lar Networks } \\
\text { (CONET) [28] }\end{array}$ & $\begin{array}{l}\text { Consumer's interest packet contains TTL } \\
\text { value to restrict the additional copies of } \\
\text { the content within the network. }\end{array}$ & $\begin{array}{l}\text { Achieves less Interest } \\
\text { Satisfaction Delay. }\end{array}$ & $\begin{array}{l}\text { This scheme causes dis- } \\
\text { connected link problem. }\end{array}$ \\
\hline $\begin{array}{l}\text { Kim } \\
\text { et al. } \\
(\mathbf{2 0 1 6 )}\end{array}$ & $\begin{array}{l}\text { Controlled Data } \\
\text { and Interest } \\
\text { Evaluation in } \\
\text { Vehicular Net- } \\
\text { works (CODIE) } \\
{[29]}\end{array}$ & $\begin{array}{l}\text { In CODIE,provider receive the interest } \\
\text { packet including number of hops. }\end{array}$ & $\begin{array}{l}\text { Author's have mini- } \\
\text { mized interest flooding } \\
\text { and Interest Satisfaction } \\
\text { Delay (ISD). }\end{array}$ & $\begin{array}{l}\text { No mobility mechanism } \\
\text { is presented at reverse } \\
\text { path. }\end{array}$ \\
\hline $\begin{array}{l}\text { Bian } \\
\text { et al. } \\
(\mathbf{2 0 1 5 )}\end{array}$ & $\begin{array}{l}\text { Geo-location } \\
\text { based forward- } \\
\text { ing [52] }\end{array}$ & $\begin{array}{l}\text { Each vehicles maintains the neighbor } \\
\text { table. Which contains the updated geo- } \\
\text { locations of the neighboring vehicles. }\end{array}$ & $\begin{array}{l}\text { Achieves high reliabil- } \\
\text { ity, high success ratio, } \\
\text { low latency rate and less } \\
\text { flooding with in the net- } \\
\text { work. }\end{array}$ & $\begin{array}{l}\text { In this scheme, best for- } \\
\text { warder selection causes } \\
\text { delay because of metrics } \\
\text { selection. }\end{array}$ \\
\hline
\end{tabular}

\section{INTERSECTION BASED FORWARDER SELECTION (IBFS) ROUTING PRO- TOCOL}

This section presents our proposed protocol named as Intersection Based Forwarder Selection (IBFS) for VCCN with particular focus on BDAS. VCCN communication paradigm is based on the receiver-driven model. In other words, when consumer is interested in a required content, it broadcasts an interest packet within its vicinity. When interest packet arrives to its neighbor node, the neighbor node checks in its CS, if prefix found then sends back the content immediately to the consumer otherwise, neighboring node rebroadcasts the same interest packet within its transmission range to search a provider. If the provider is found, it generates the data packet and sends back to the consumer via selected intermediate neighbor nodes as shown in Fig. 7.

\subsection{Challenges of Traditional Routing in VCCN}

We present different scenarios of interest broadcast storm in a VCCN in the following section. This will determine the traditional routing challenges in the VCCN. 


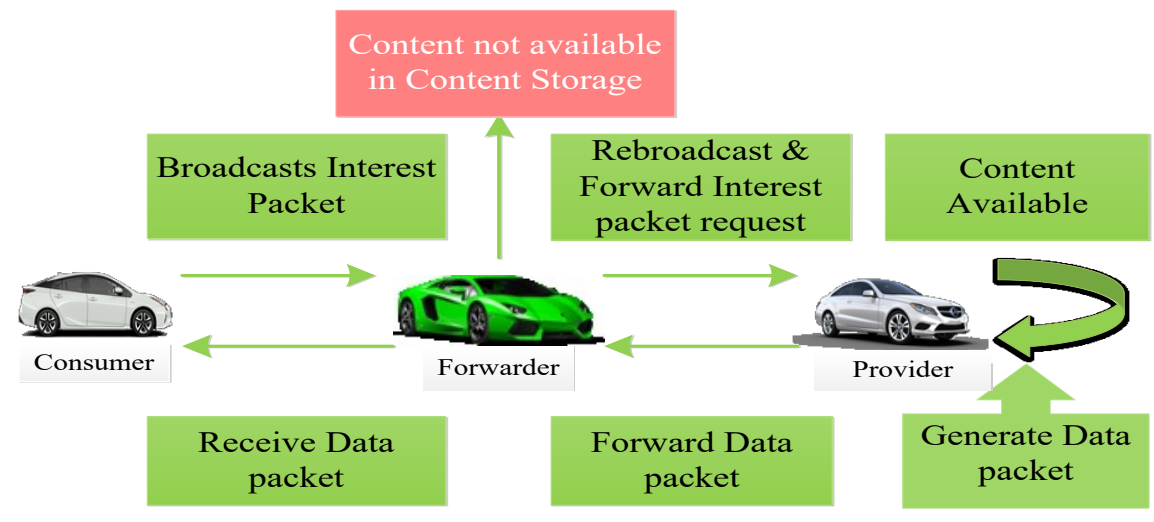

Figure 7: Traditional VCCN Routing Architecture

\subsubsection{Interest Broadcast Storm}

We have considered an interest broadcast scenario where a consumer sends an interest packet to its neighboring nodes within its vicinity. Upon receiving the interest packet, all neighboring nodes further check the particular content in its CS. In case of no match, all neighbor nodes become a forwarder vehicle and rebroadcast the interest packet within its vicinity in order to search a provider as shown in Fig. 8.

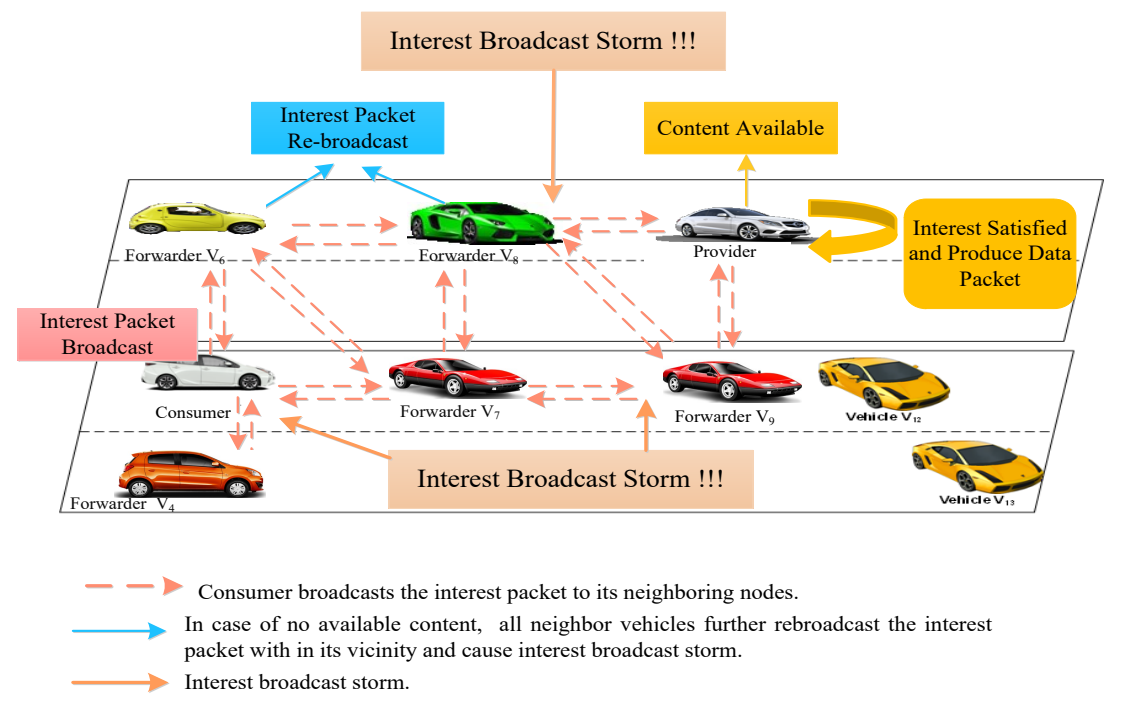

Figure 8: All neighbor's vehicle rebroadcast the interest packet in its vicinity and cause interest broadcasting storm

When all forwarder vehicles disseminate the interest packet to its neighbor nodes to find a provider with in its vicinity who can satisfy the interest, this results in a interest broadcast storm. This broadcast storm creates congestion in the entire network [53]. 


\subsubsection{Disconnected Link Problem}

In this scenario, we have considered the speed ranging $70-90 \mathrm{~km} / \mathrm{hr}$ among different vehicles because compatible speed have been considered in RUFS [27]. When a consumer is interested in some desired content, firstly, the consumer shares all information like hop-count or Interest Satisfaction Ratio (ISR) with its neighboring node as shown in Fig. 9 [27].

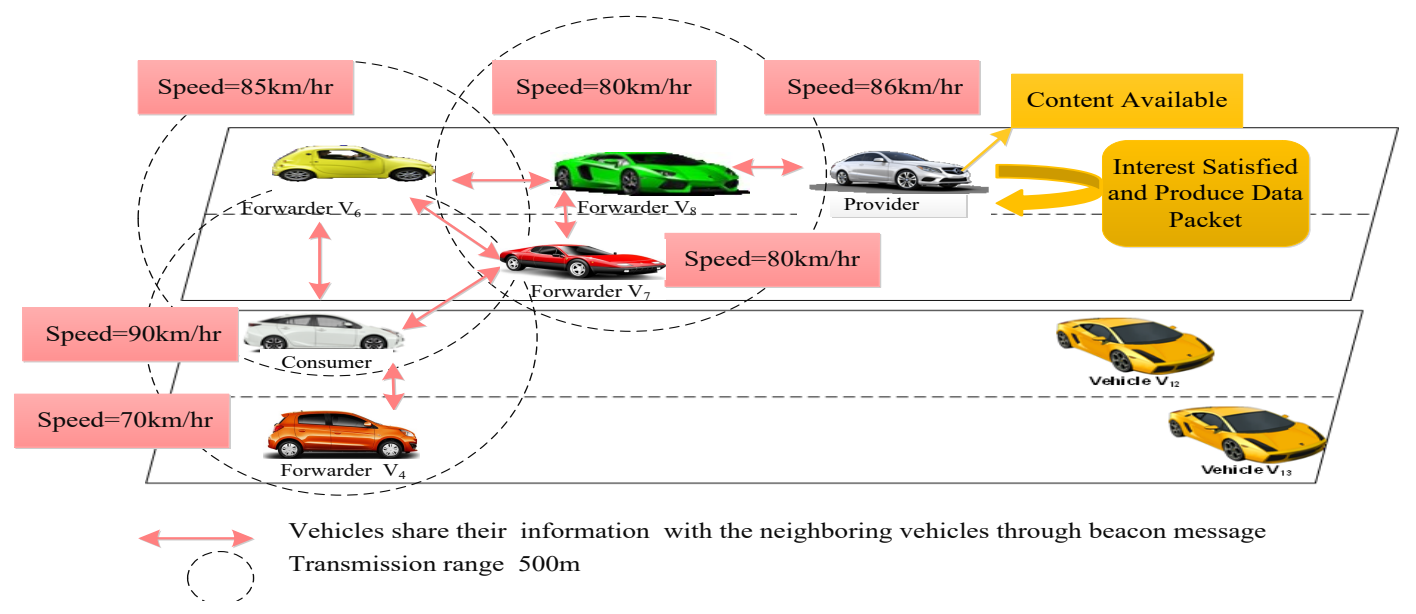

Figure 9: Consumer shares all information with its neighboring vehicles with in the vicinity.

After sharing information, the consumer selects the best forwarder (i.e., a neighbor) vehicle on the basis of hop-count and high ISR. In case, of no content is available in the CS of the neighbor, the neighbor vehicle performs three actions, i.e., (i) shares all information with its next neighboring nodes within the vicinity; (ii) rebroadcasts the same interest packet and (iii) selects another best forwarder vehicle on the basis of lesser hop-count and high ISR as shown in Fig. 10.

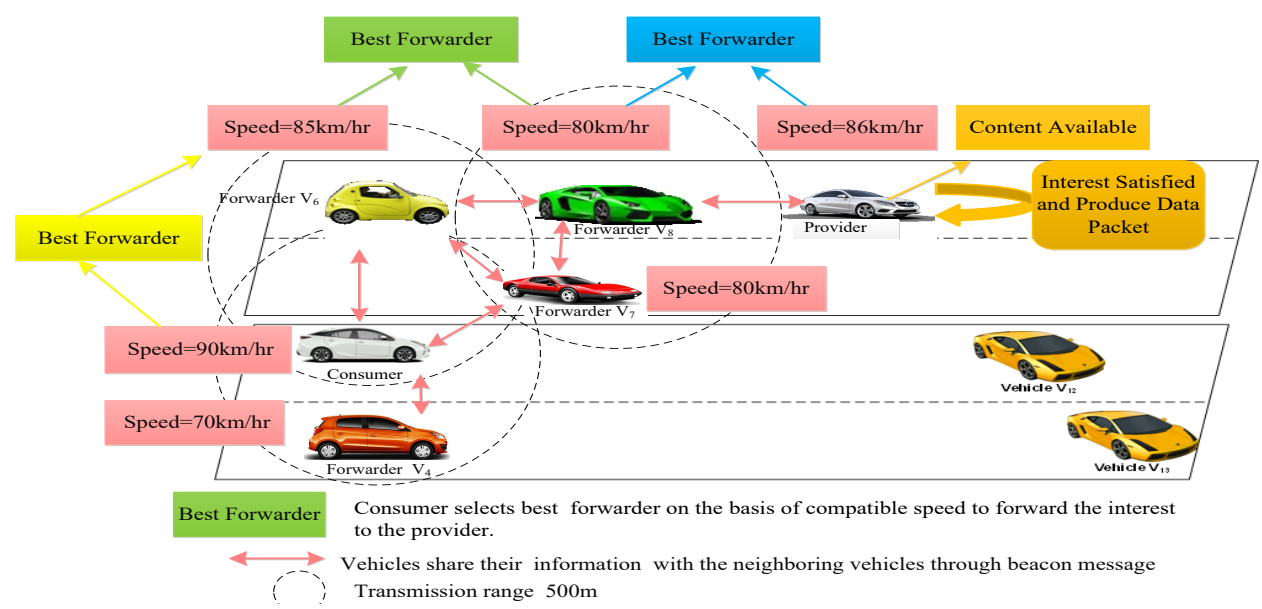

Figure 10: Consumer selects the best forwarder to mitigate the interest broadcast storm. 
In case, if the content is available in the CS of the first neighbor, this neighbor becomes best forwarder and generates the data packet. Furthermore, the response data packet is sent back to the consumer via selected forwarder vehicles [26]as shown in Fig. 11.

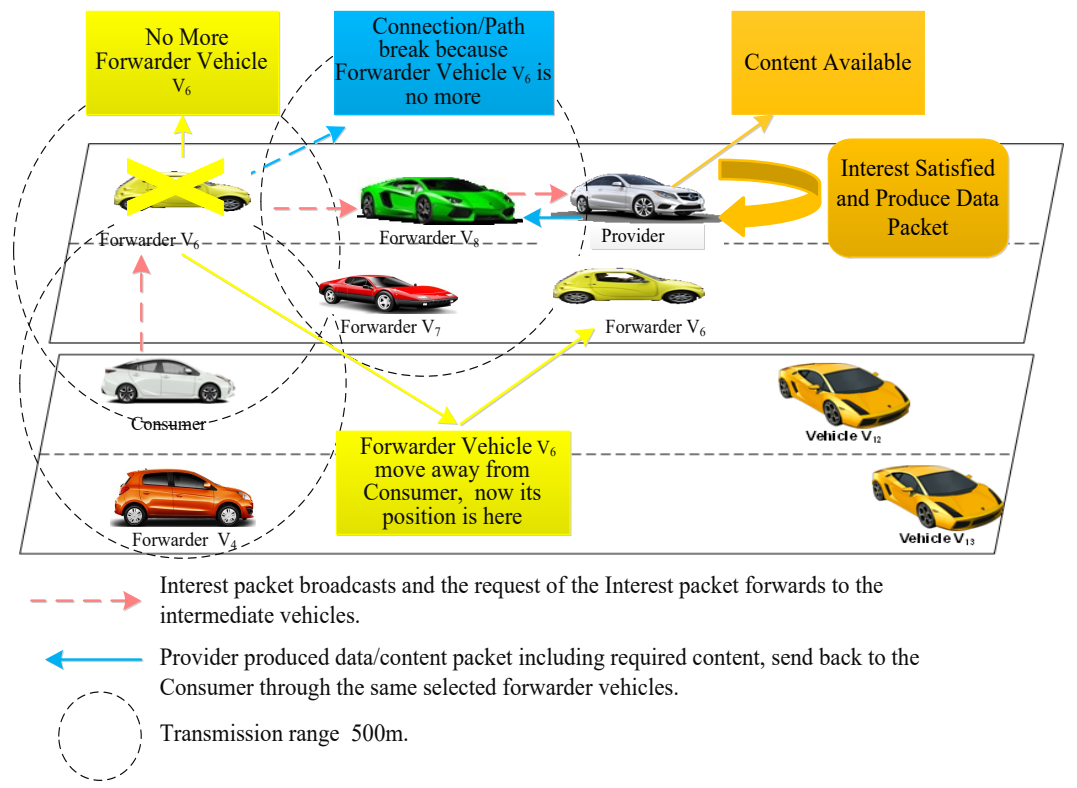

Figure 11: Disconnected link problem.

It can be observed that the interest broadcast storm and the disconnected link problems can result in the degraded network performance. The proposed IBFS routing protocol for VCCN efficiently deals with these problems. In the subsequent sections, the design details of the proposed IBFS protocol are discussed.

\subsection{IBFS Protocol Data Structures}

In the proposed IBFS routing protocol, each vehicle maintains two types of data structures which are described below:

- VIL (Vehicle Information List)

- NIT (Neighbor Information Tale)

\subsubsection{VIL}

Each vehicle has its own IBFS performance metrics e.g., intersection Information (trajectory) and velocity etc. We take multiple intersections like Intersection 1, Intersection 2, and Intersection 3 to reach a destination. According to different international standards of speed, we have considered speed ranging from 70-90 km/hr. This also ensures the maximum connection among different vehicles [27]. Each vehicle must exchange the VIL with its neighboring node in the range through a beacon a message. The VIL contains trajectory information and the vehicular speed as shown in Fig. 12. 


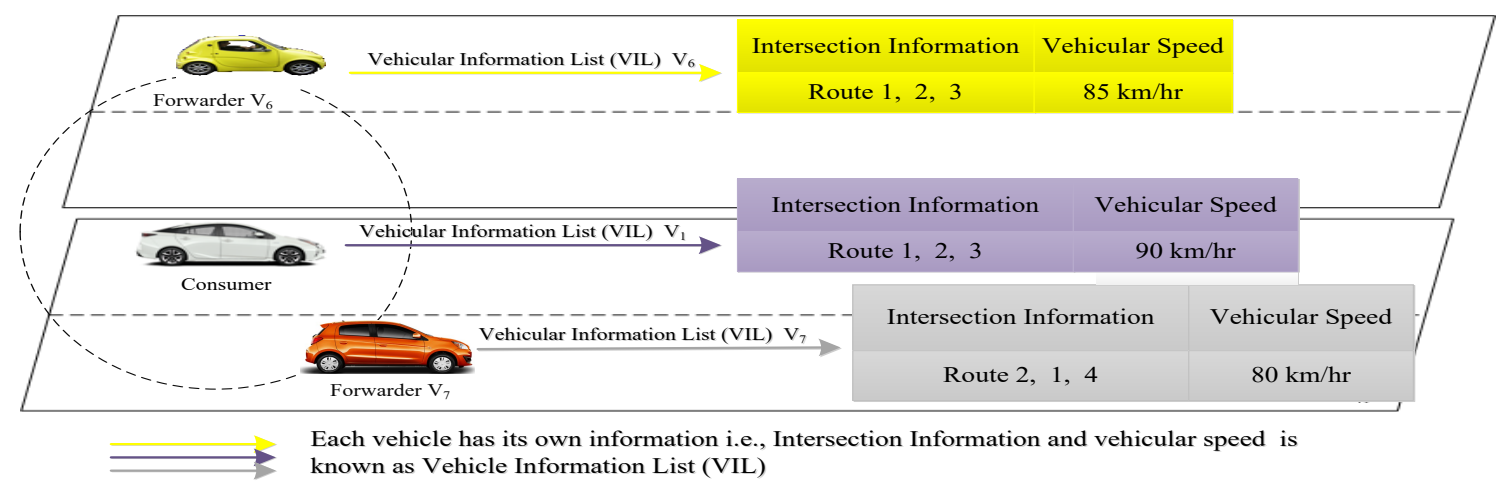

Figure 12: Each vehicle has its own IBFS parameters

\subsection{2. $N I T$}

NIT contains the information of the data/content packet currently satisfied by its neighbor vehicle. All vehicles exchange their information with neighbors such as vehicle ID, trajectory information, vehicular speed and hop-count. We use Vehicle-ID to give a unique identity to each vehicle, trajectory information is used to build a connection for longer period. We use one-hop to make the experiments more realistic. However, hop-count may change at any time as it depends on the transmission range. If transmission becomes greater, the number of hops will be higher. The information received from the neighbors is saved in the NIT as shown in the Fig. 13. The maintenance of the NIT at each node helps in identifying the appropriate routing decisions.

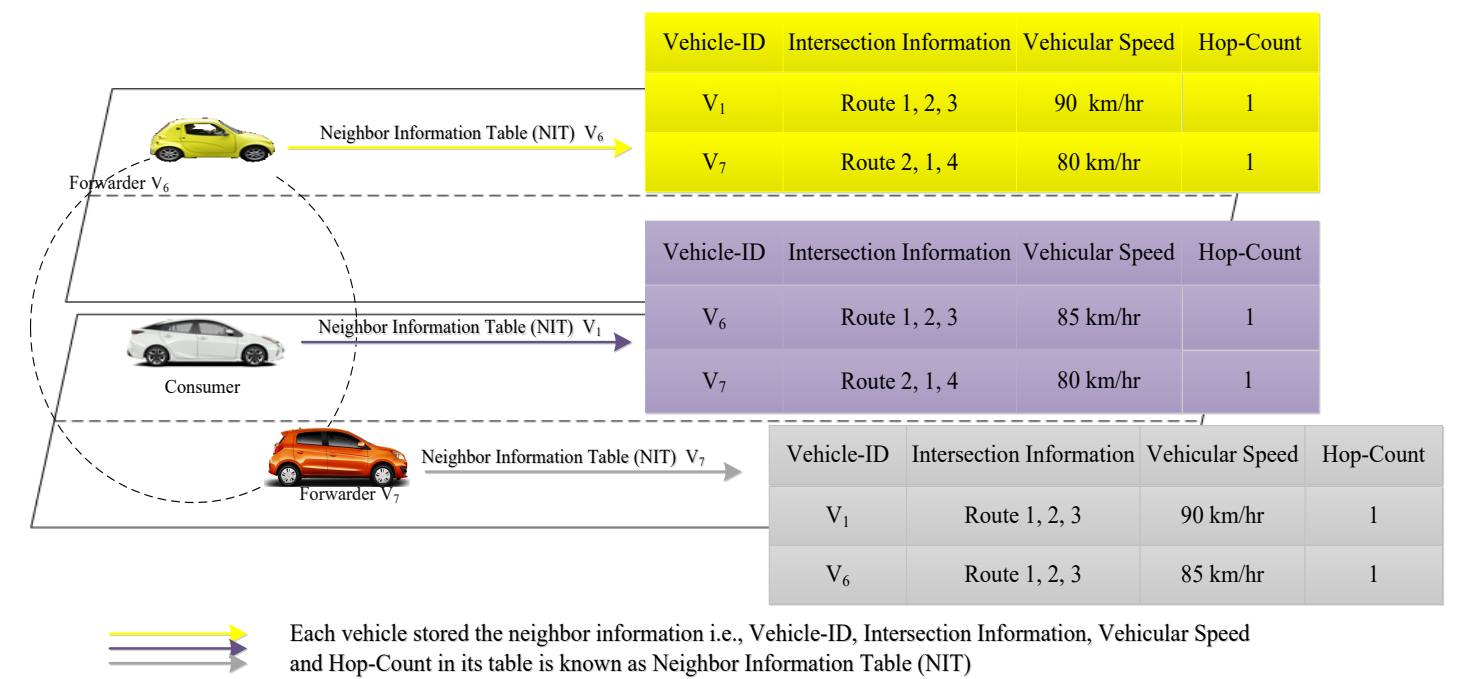

Figure 13: Each vehicle stores the information of its neighbor vehicles in the NIT 


\subsection{IBFS Routing Phases}

IBFS routing protocol operates in two phases. We discuss these phases in following section:

\subsubsection{Intersection Information Sharing (IIS) Phase}

In this phase, every vehicle has its own VIL and communication range (500m)[54]. When a consumer is interested in some content, the consumer vehicle sends the VIL through a beacon message to its one-hop neighborâĂŹs node (i.e., intermediate vehicles). The beacon message format is shown in Fig. 14. We have considered one-hop neighbor node to achieve minimum number of hop but it can be changed with respect to the real environment. In beacon message format, we have considered three different fields are: (i) Consumer Vehicle-ID: This is used to give the unique identity to each vehicle. The size is 1 Byte. To accommodate different vehicle-IDs in 1byte, we have tested 10 numbers of vehicles. (ii) Intersection Information: A route is called an intersection. This is 3Bytes field. We use multiple intersection points to reach a destination. For example, intersections 1,2 , and 3 provide the reliable communication and ensure maximum transmission of interest and data packet. (iii) Vehicular Speed: This is a 2Byte field and uses different speeds ranging from $70-90 \mathrm{~km} / \mathrm{hr}$. The vehicle speed ensures the maximum connection in less time. We have multiple consumers to transmit the interest packets and the interest packet requests are satisfied by multiple providers in the network. To make the proposed protocol flexible, we use the additional bytes to accommodate different vehicle-IDs with its Intersection Information and Vehicular speed. The beacon message format is shown in Table. 2 .

Table 2: Beacon Message Format

\begin{tabular}{|l|l|l|}
\hline Consumer Vehicle-ID (Byte) & Intersection Information (Byte) & Vehicular Speed (Byte) \\
\hline
\end{tabular}

All neighboring vehicles store information in its NIT as discussed in previous section. In a response, a neighbor node sends its information to the consumer and the consumer stores the neighbor 's information in its own NIT. The stored information helps the consumer to choose the intermediate (neighbor) node as a best forwarder or a relay to form optimized communication links within the available transmission range as shown in Fig. 14. This makes communication more reliable because of most of the nodes do not do not take part in the forwarding of interest packet and other related operations. This also minimizes the interest broadcsst storm in the entire network.

\subsubsection{Interest/Data Packet Forwarding Phase}

After IIS phase, a consumer broadcasts an interest packet and chooses a single intermediate (neighbor) vehicle as a best forwarder/relay whose trajectory information matches and the velocity is compatible with the consumer. This scenario is shown in Fig. 15.

The neighboring vehicles whose trajectory information are match they become the best forwarder otherwise in case of do not match, they discard the interest packet. In this way, the proposed IBFS mitigates the interest broadcast storm and provides stability to the network because nonparticipation of the rest of neighboring vehicles in the interest forwarding. This scenario is depicted in Fig. 16. 


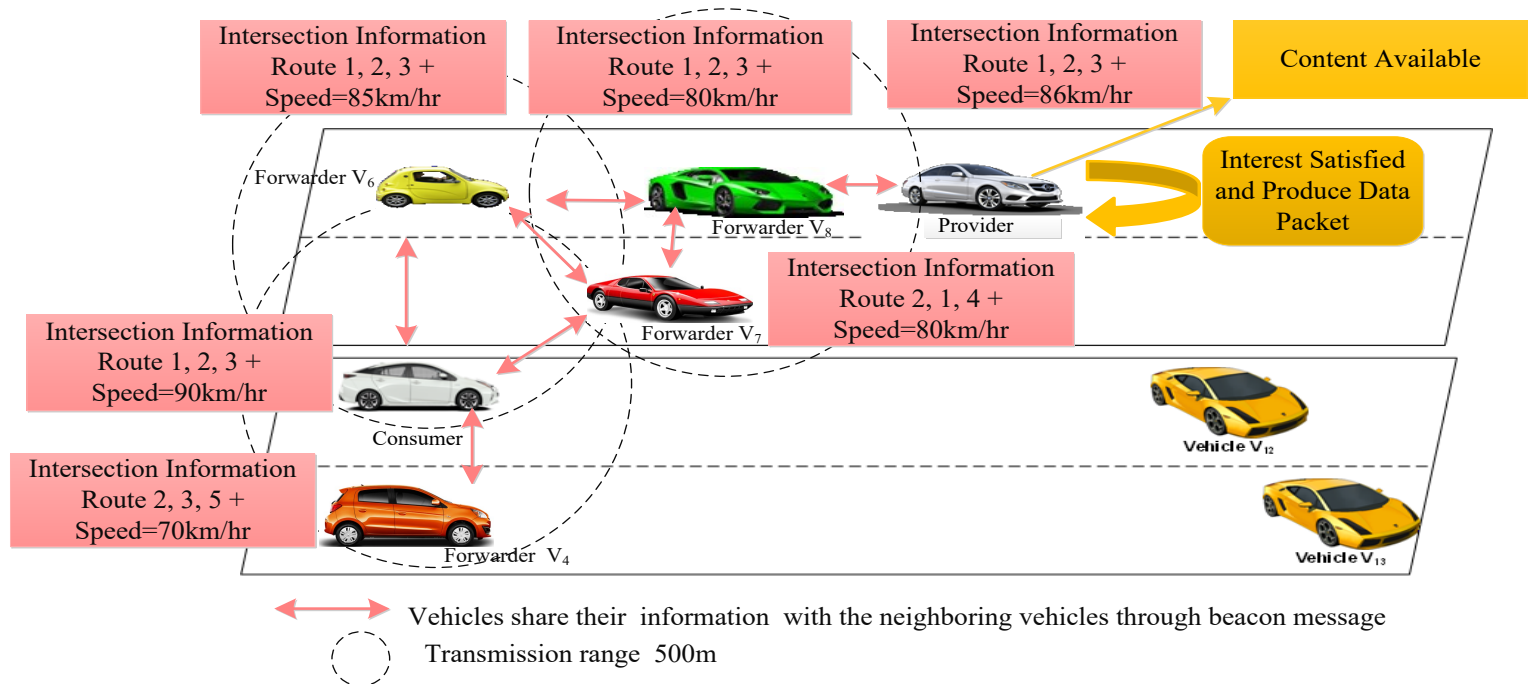

Figure 14: Consumer shares all information with its neighboring vehicles through beacon message.

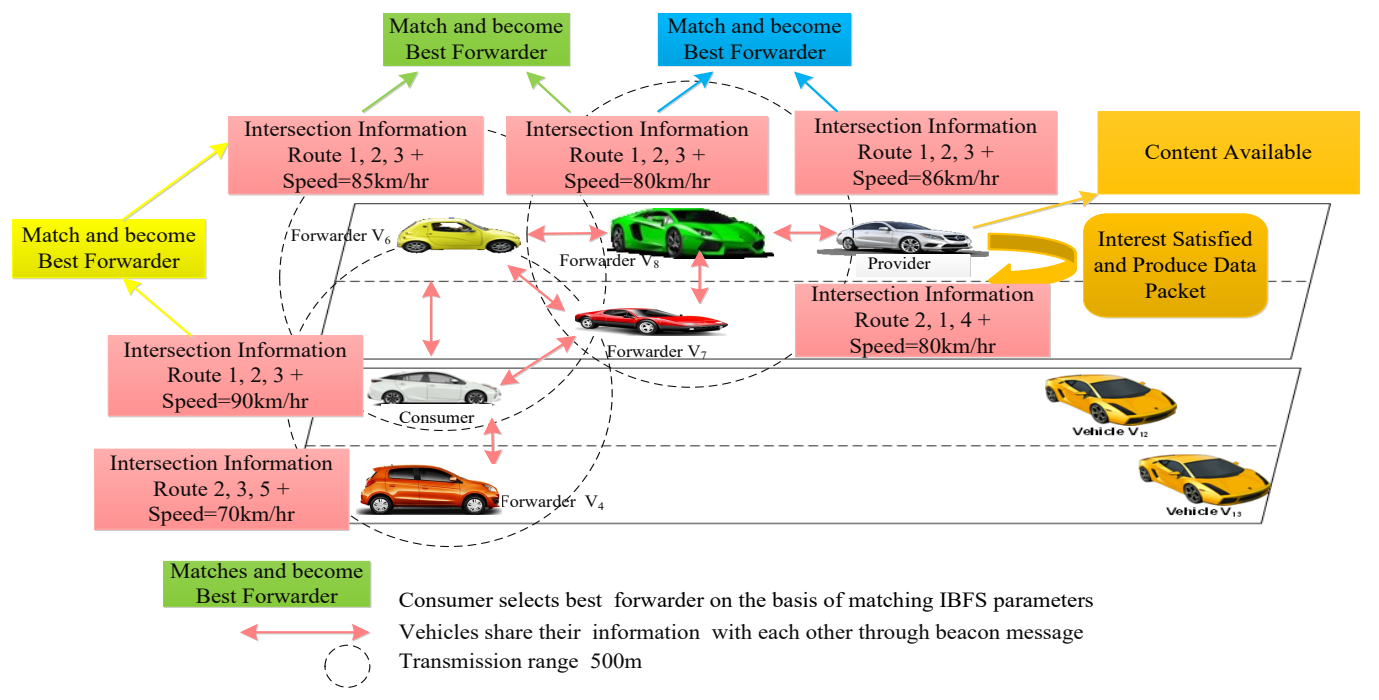

Figure 15: Consumer IBFS parameters matches with the neighboring vehicles and becomes a best forwarder.

In Algorithm 1, each vehicle V has its own VIL called $V I L_{\text {info }}$. When a consumer $\mathrm{C}$ is interested in some content, it sends its $V I L_{\text {info }}$ to its neighboring nodes called $N_{\text {nodes }}$. During the exchange of information, each vehicle creates NIT in order to store the information of the neighbor vehicles. After exchanging information, the consumer $\mathrm{C}$ broadcasts an interest packet called $I_{\text {interestpacket }}$ to choose the best forwarder called $B_{\text {forwarder }}$. If consumer's VIL called VIL consumer (trajectory and velocity) information matches the neighbor vehicle VIL (which is called $V I L_{\text {neighbour }}$ ) which is already stored in the $V I L_{\text {consumer }}$, the C selects the neighbor node as the $B_{\text {forwarder }}$ and forwards the interest packet. Otherwise, if the information is not matched with the $V I L_{\text {consumer }}$, the neighbor nodes discard the $I_{\text {interestpacket }}$. 


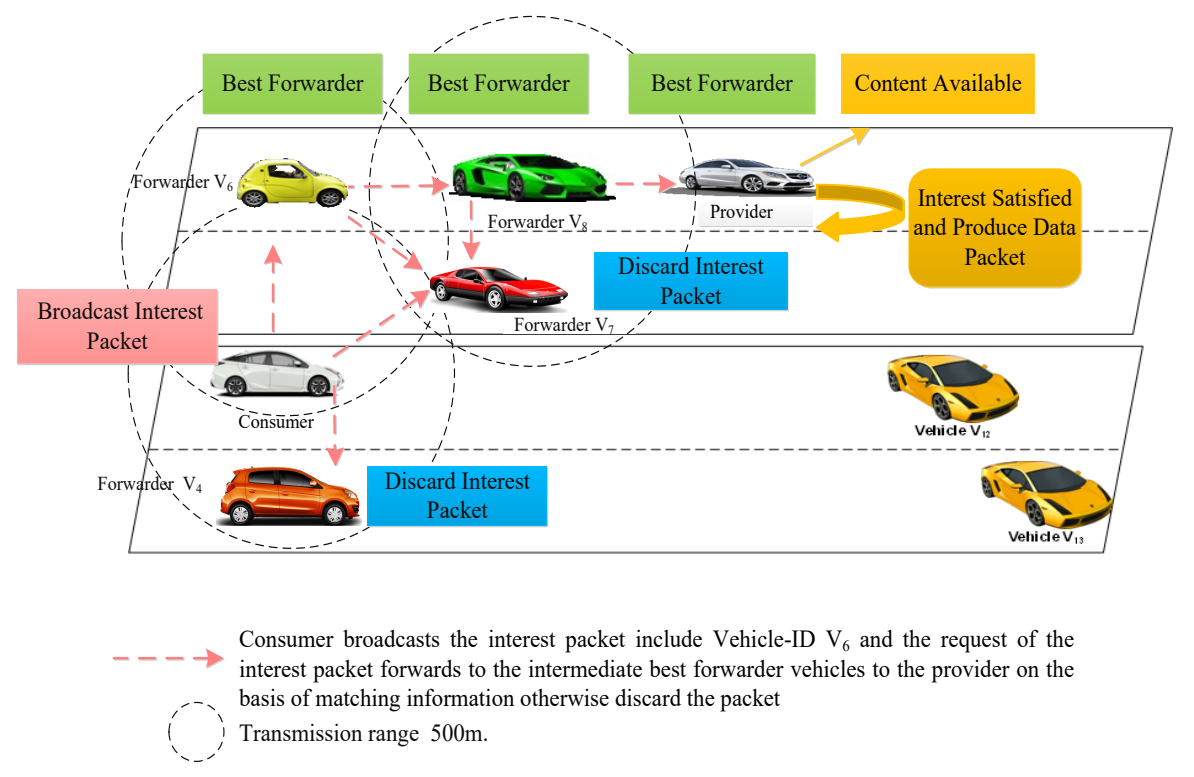

Figure 16: Forwarder/Neighbour vehicles discard packet formation due to mismatch and best forwarder formation due to matching IBFS parameters

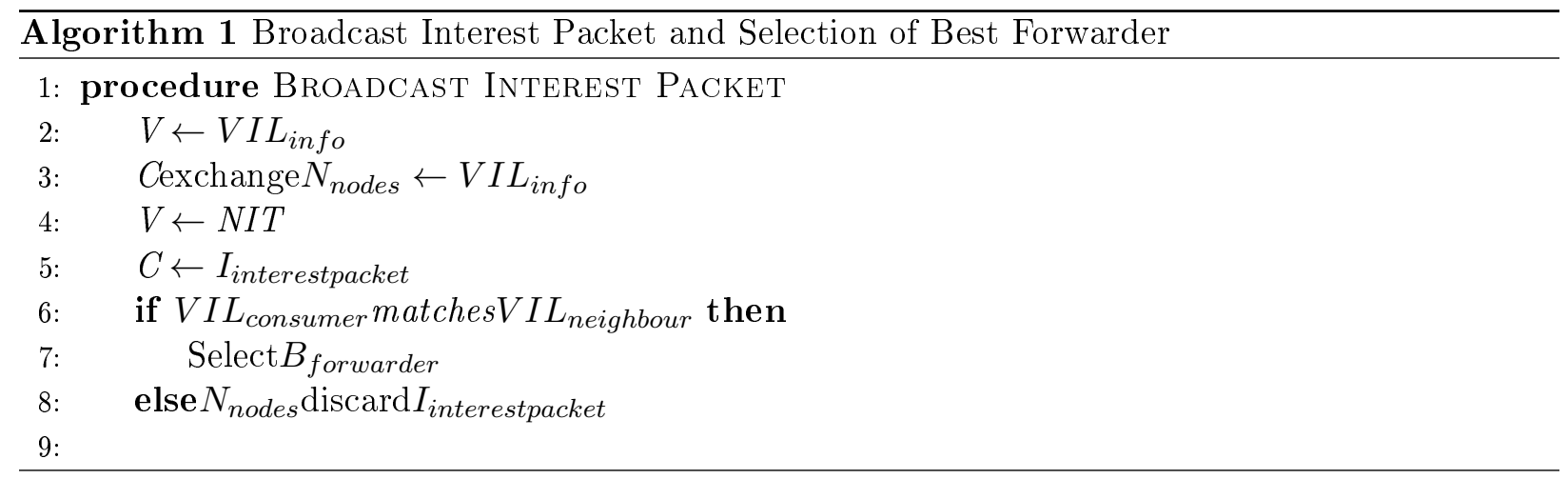

Algorithm 2 describes the behavior of the $B_{\text {forwarder }}$. The $B_{\text {forwarder }}$ receives the interest packet $I_{\text {interestpacket }}$ from the $\mathrm{C}$ and checks the required content named as $C_{\text {content }}$ int in its CS. If $C_{\text {content }_{\text {int }}}$ is available, this $B_{\text {forwarder }}$ becomes the content forwarder. We name it as $C S_{\text {forwarder }}$. The $C S_{\text {forwarder }}$ satisfies the request and generates the data packet called $P_{\text {datapacket }}$ and sends back to the C. If the request could not be satisfied, two functions are performed: i) this $I_{\text {interestpacket }}$ is stored in the local Pending Interest Table (PIT) and ii) using the FIB, the $I_{\text {interestpacket }}$ is forwarded to the those neighbors whose trajectory and velocity are compatible.

Interest packet is forwarded to the provider via selected forwarder vehicles. In response, provider generates the data/content packet including required content, content-ID and hop-count. The data is sent back to the consumer through same selected forwarder/relay vehicles as shown in Fig. 17. The performance metrics such as intersection information, vehicular speed and the hop-count used by the proposed IBFS, achieves successful delivery of the data packet to the consumer. 

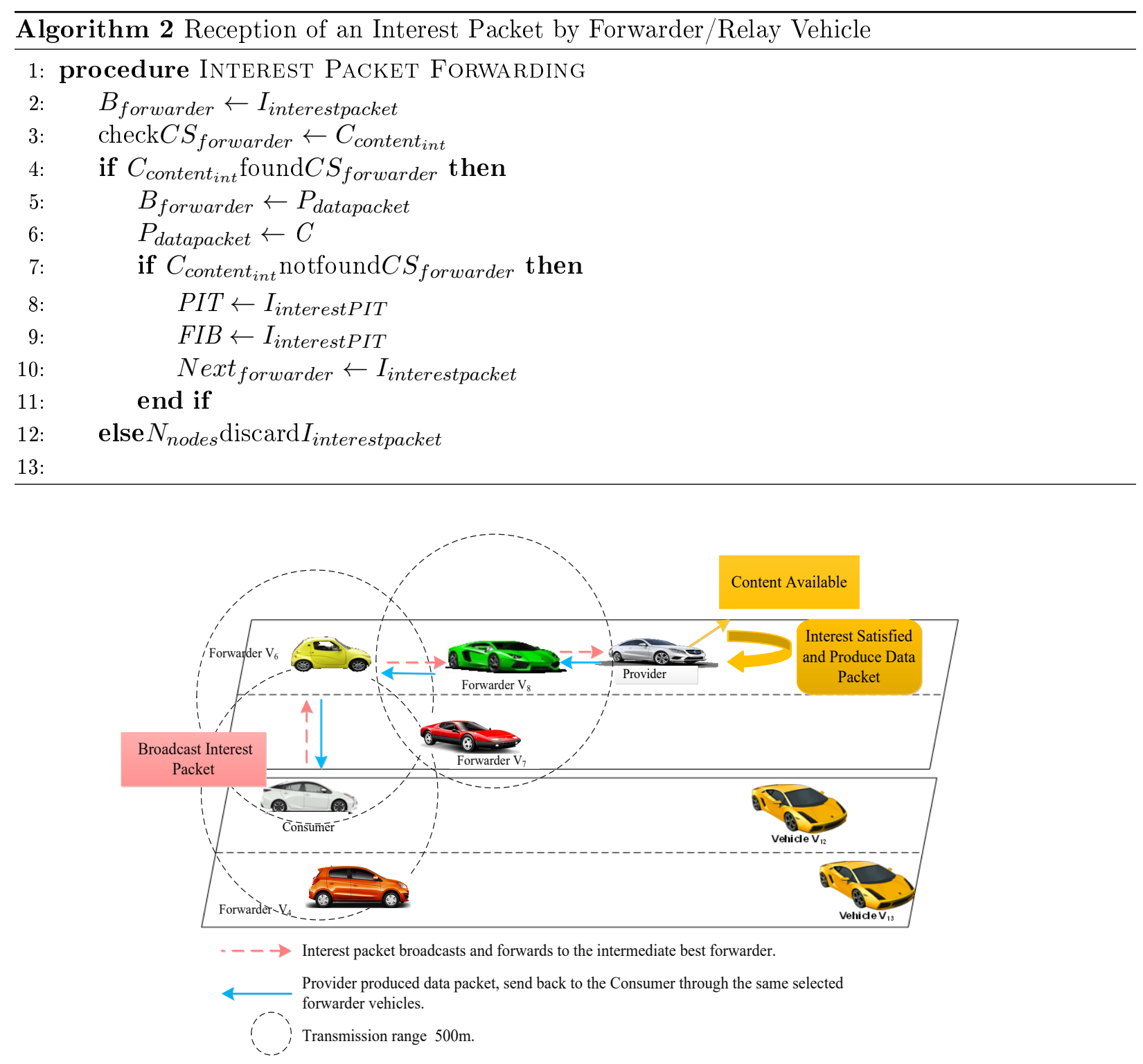

Figure 17: Consumer broadcasts the packet to the provider through best forwarder vehicles and provider send the data packet as a reply via selected forwarder vehicles.

Now, the Algorithm 3 is discussed which shows the receipt of the data packet by the forwarder/rely node. At reverse path, when $B_{\text {forwarder }}$ receives the $P_{\text {datapacket }}$ form the provider, it checks the corresponding PIT entry (named as PIT content $_{\text {int }}$ along with the $C_{\text {content }_{\text {int }}}$. Furthermore, it checks the value of the hop-count of the provider in the $P I T_{\text {content }_{\text {int }(h)}}$. If the hop-count of the provider vehicle equals the hop-count of the $B_{\text {forwarder }(h)}$, the forwarder vehicles decrement the value of the hop-count i.e., $B_{\text {forwarder }(h-1)}$ and removes the entry from the PIT and forwards this content in to the CS of the $B_{\text {forwarder. }}$. Whenever the content becomes available in the CS $B_{\text {forwarder }(h)}$, it forwards the content to the C. 


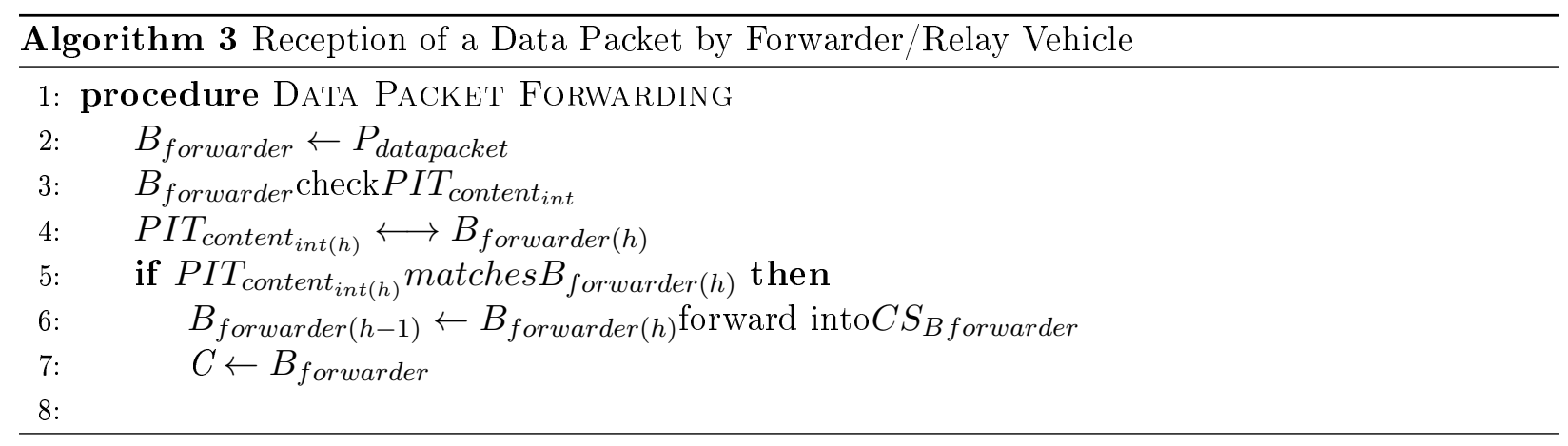

\subsection{IBFS Mathematical Model}

In IBFS, each vehicle selects the best forwarder vehicle on the basis of IBFS metrics i.e., intersection information, vehicular speed, and hop-count within its transmission range. The problem is that the above-mentioned metrics have different units like Intersection Information is measured in natural numbers, vehicle speed is measured in $\mathrm{km} / \mathrm{hr}$ and the hop-count is a natural number. We use topsis model [55] and the multiple criteria decision method (MCDM). We consider a scenario of forwarding phase and apply topsis model on this scenario. Suppose, a consumer's vehicle i.e., $V_{1}$ sends a request of an interest packet to any forwarder vehicle say $V_{6}$ on the basis of closely matched intersection trajectory (e.g., Route $1,2,3$ ). The $V_{6}$ selects next forwarder vehicle say $V_{8}$ on the basis of its intersection trajectory and compatible speed. The $V_{8}$ (also called a relay vehicle) selects the provider vehicle, say $V_{10}$ to forward an Interest packet. The $V_{10}$ replies back to the $V_{1}$ with the data packet through the $V_{8}$ and $V_{6}$. See Figure 6 which represents the Interest/Data Packet forwarding phase between a consumer and the provider. If the Intersection Information of the vehicle $\left(V_{i}\right)$ matches with the consumer vehicle, we consider the two parameters i.e., vehicular speed and hop-count with the help of judgmental value 1, otherwise, if there is no match, we ignore the other parameters and the judgmental value becomes 0 . The equation to select the best forwarder vehicle is as follow:

$V_{m}=\left(\begin{array}{c}\text { IntersectionIn formationtrajectorymatches }=1 \\ \text { IntersectionIn formationtrajectorynotmatches }=0\end{array}\right)$

Where $\mathrm{i}=1,2,3,4 \ldots . \mathrm{m}$.

Step I. The Vehicle $V_{m}$, where $\mathrm{i}=1,6,8 \ldots . . \mathrm{m}$ maintains the sequence of neighbouring vehicle intersection information for required content $I C_{n}$ as :

$$
X_{m n}=\left(\begin{array}{ccc}
V_{1,1} & V_{1,2} & V_{1,3} \\
V_{2,1} & V_{2,2} & V_{2,3} \\
V_{3,1} & V_{3,2} & V_{3,3} \\
\vdots & \vdots & \vdots \\
V_{m, 1} & V_{m, 2} & V_{m, n}
\end{array}\right)
$$

$X_{m n}$ is a neighbour vehicle $V_{m}$ criterion $C_{n}$.

Step II. The parameters of $C_{n}$ are different and required to be normalized decision matrix scale. The normalized value of $P_{i j}$ is defined as: 


$$
P_{i j}=\frac{x_{m n}}{\sqrt{\sum_{m=1}^{i} x_{m n}^{2}}}
$$

Step III. $W_{n}$ Is the preference values assigned to each property $C_{n}$, so weighted normalized $P_{i j}$ is calculated as:

$\mathrm{R}_{m n}=W_{n} * P_{m n}$ Wherem $=1 \ldots i \& n=1 \ldots 3$

$\mathrm{W}_{n}$ Is the weight of the $m^{\text {th }}$ criteria, so is equally distributed for all criteria so

$$
\sum_{m=1}^{j} W_{n}=1
$$

Step IV. For each $C_{n}$, the positive ideal solution $I^{+}$and the negative ideal solution $I^{-}$are:

$$
\begin{aligned}
& I^{+}=\left[\max R_{m n} \mid n \epsilon N\right],\left[\operatorname{minRmn} \mid n \epsilon N^{\prime}\right] \mid m=1,2,3 \ldots i=\left[\mathrm{R}_{1 n}+, R_{2 n}+, R_{3 n} \ldots+, R_{i n}\right] \\
& I^{-}=\left[\min R_{m n} \mid n \epsilon N\right],\left[\max R m n \mid n \epsilon N^{\prime}\right] \mid m=1,2,3 \ldots i=\left[\mathrm{R}_{1 n}+, R_{2 n}+, R_{3 n} \ldots+, R_{i n}\right]
\end{aligned}
$$

Step V. Every neighbor vehicle with the most ideal solution $D_{i}^{+}$the worst vehicle solution $D_{i}^{-}$is calculated by using Equation 4 and 5 .

$$
\begin{aligned}
\left(D_{i}^{+}\right) & =\sqrt{\sum_{m=1}^{j} I^{+}-R_{m n}{ }^{2}} \\
\left(D_{i}^{-}\right) & =\sqrt{\sum_{m=1}^{j} I^{-}-R_{m n}{ }^{2}}
\end{aligned}
$$

Where $\mathrm{m}=1 \ldots . \mathrm{i} \quad \mathrm{n}=1 \ldots . .3$

Step VI. The neighbour vehicle in is calculated with respect to the positive ideal solution by using Equation 6 and 7.

$$
B_{i j}=\frac{D_{i}^{-}}{D_{i}^{+}+D_{i}^{-}}
$$

On the basis of the above discussed IBFS routing phases, algorithms and mathematical model,we have concluded, By applying topsis[55] model, IBFS forwarder scheme performs very well both in highway and urban scenario in VCCN paradigm. 


\section{PERFORMANCE EVALUATION}

The IBFS routing protocol is implemented using the Network Simulator (NS-2) [56] in a VANET environment with different number of vehicle nodes i.e., 20-45. These vehicle nodes have already been considered for the evaluation in RUFS [27]. All vehicle nodes are deployed randomly using Random Direction Mobility Model. In real environment, we can not control the direction of the mobile vehicles. To make our simulation more realistic, we use the mobility model. This provides different direction of the vehicles while taking into account the mobility and random velocity of the vehicles. Furthermore, the mobility model ensures maximum connections. The velocity of the vehicles is set between $70-90 \mathrm{~km} / \mathrm{hr}$ (randomly generated).

The IBFS protocol functions on MAC layer IEEE 802.11p for accessing the wireless channel in a vehicular environment.IEEE 802.11p is also known as WAVE (Wireless Access for Vehicular Environments) and DSRC (Dedicated Short Range Communication) [57]. The main objective of 802.11p is to improve traffic efficiency. The network formed by 802.11p is known as VANET. In IBFS routing protocol, all vehicles are equipped with a Global Positioning System (GPS) to communicate with each other and to provide the information related to the mobility within the whole network. We use V2V communication paradigm in our proposed IBFS routing protocol. In vehicular environment, Wireless Access in Vehicular Networks (WAVE) and Dedicated ShortRange Communications (DSRC) achieves a transmission range 500m [57]. All the parameters of the simulation are summarized in Table 3 .

Table 3: Properties of the Simulation Parameters

\begin{tabular}{|l|l|}
\hline Network Simulator & NS-2 \\
\hline Number of Vehicle Nodes & $20,25,30,35,40,45$ \\
\hline Area of Simulation Size & $10000 \mathrm{~m}^{*} 10000 \mathrm{~m}$ \\
\hline Transmission Range & $500 \mathrm{~m}$ \\
\hline Packet Size & 1024 bytes \\
\hline Propagation Model & Two Way Ground/Propagation \\
\hline MAC Layer & IEEE $802.11 \mathrm{p}$ \\
\hline Transmission Power & $0.0091 \mathrm{~mW}$ \\
\hline
\end{tabular}

We considered two different scenarios for our simulation experiments, i.e., urban and highway. Both scenarios are implemented in a VANET environment by using SUMO. The NS-2 is used to implement both the scenarios. We take average of thirty independent simulation iterations.

\subsection{IBFS Performance Parameters}

To evaluate the performance of IBFS and RUFS [27] routing protocol, the performance metrics are: Packet Delivery Ratio (PDR), Packet Loss Ratio (PLR), Interest Satisfaction Ratio (ISR), and Average End-to-End Delay. We discuss the above parameter in detail one by one as follows.

\subsection{Packet Delivery Ratio(PDR)}

Packet Drop Ratio (PDR) is an important parameter for analyzing the performance of different networks. PDR shows the percentage of successful data packets, which are successfully delivered 
at the end of the transmission. The fundamental goal of every routing protocol is to achieve higher delivery ratio. The following formula is used to calculate Packet Delivery Ratio.

$\mathrm{PDR}=(\text { Number of Received Packets/Number of Send Packets })^{*} 100$.

\subsection{Packet Loss Ratio(PLR)}

Packet Loss Ratio (PLR) is another important parameter for checking the performance of different networks. It shows the percentage of dropped data packets which are lost during the transmission. Packet loss is actually calculated from the Packet Delivery Ratio (PDR) which is also an important performance parameters. Following formula is used to calculate packet loss ratio.

PLR $=100-$ (Number of Received Packets/Number of Send Packets)*100.

\subsubsection{Average End-to-End Delay}

The average delay is the time (measured in second or millisecond) taken from source to the provider travelled by the data packet in a network. The time when the packets are transmitted from the source is noted and when it arrives at the destination (provider) and then its arrival time is calculated. The following formula is used to calculate the Average Delay.

Delay $=$ Received time-Transmit time

Average End-to-End Delay = Sum of all Packets Delay/ Total Number of Received Packets.

\subsubsection{Interest Satisfaction Ratio(ISR)}

Interest packet Ratio is defined as follows.

ISR $=$ Total Interest response Received / Total Interest Generated.

\subsection{Performance Result and Discussion}

In this section, the experimental results of the proposed IBFS and RUFS for highway and urban scenarios are discussed.

\subsubsection{Highway Scenario}

In this section, we will consider the experimental results of Packet Delivery Ratio in highway scenario are:

4.4.1.1 Packet Delivery Ratio (PDR)

The Table 4 summarized the performance comparison of IBFS and RUFS in terms of PDR.

Table 4: Packet Delivery Ratio Comparison in Highway Scenario

\begin{tabular}{|l|l|l|}
\hline Number of Nodes & IBFS & RUFS \\
\hline $\mathbf{2 0}$ & $76 \%$ & $16 \%$ \\
\hline $\mathbf{2 5}$ & $79 \%$ & $21 \%$ \\
\hline $\mathbf{3 0}$ & $84 \%$ & $24 \%$ \\
\hline $\mathbf{3 5}$ & $86 \%$ & $29 \%$ \\
\hline $\mathbf{4 0}$ & $88 \%$ & $40 \%$ \\
\hline $\mathbf{4 5}$ & $90 \%$ & $40 \%$ \\
\hline
\end{tabular}

In IBFS, the PDR is higher because of the more packets are successfully delivered due to stable link transmission. In RUFS, when any forwarder vehicle moves away from the consumer, the absence of any other forwarder causes disconnected link problem. In IBFS, the quality of the stable 
state of the transmission link in terms of Intersection (i.e., trajectory) and velocity is measured and both the trajectory and the velocity are used as IBFS parameters to forward data through selected forwarder vehicles. This means that there are more chances of receiving a data packet at provider resulting in reliable packet routing. In the highway scenario, more number of packets are delivered and received successfully at the provider and the PDR is higher than RUFS as shown in Fig. 18 .

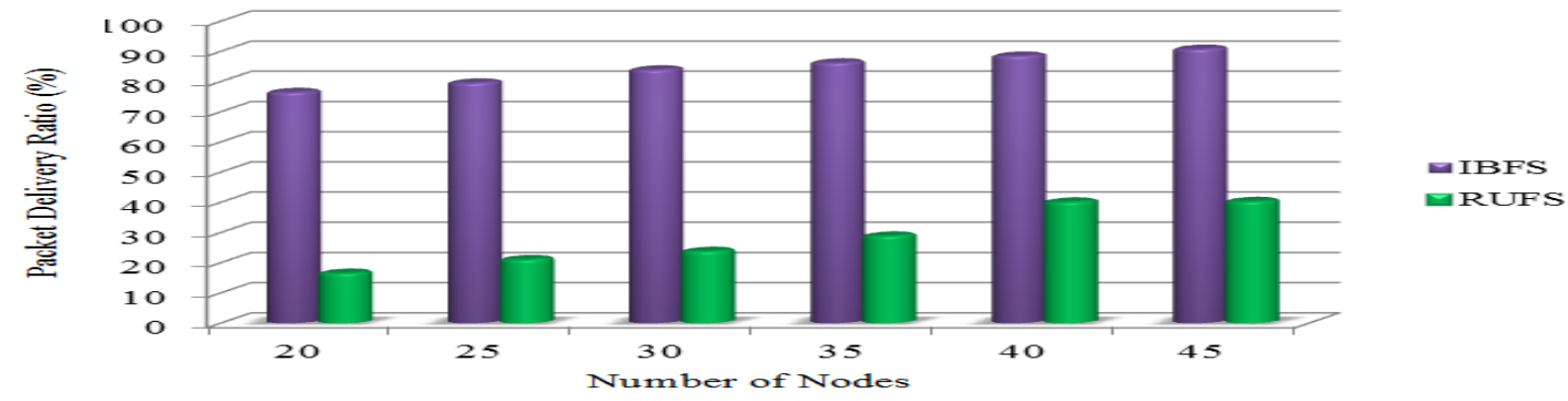

Figure 18: Packet Delivery Ratio (PDR) vs Number of Vehicular Nodes in Highway Scenario

In this section, we will consider the experimental results of Packet Loss Ratio in highway scenario are:

\subsubsection{Packet Loss Ratio (PLR)}

We analyze the number of lost packets due to the disconnected link problem and investigate that most of the time, the poor quality of the transmission of data packets results in higher PLR. It raises the probability of data being dropped due to instable link connections. In IBFS, the quality of the transmission link is measured for every link and is used as a major performance metric to select the best forwarder. Through this, we reduce the chances of instable and poor data packet forwarding link. This results in the IBFS having lower ratio of dropped packets when compared with RUFS as summarized in Table 5.

Table 5: Packet Loss Ratio Comparison in Highway Scenario

\begin{tabular}{|l|l|l|}
\hline Number of Nodes & IBFS & RUFS \\
\hline $\mathbf{2 0}$ & $23.5451 \%$ & $83.4443 \%$ \\
\hline $\mathbf{2 5}$ & $20 \%$ & $79 \%$ \\
\hline $\mathbf{3 0}$ & $16 \%$ & $76 \%$ \\
\hline $\mathbf{3 5}$ & $14 \%$ & $71 \%$ \\
\hline $\mathbf{4 0}$ & $11 \%$ & $59 \%$ \\
\hline $\mathbf{4 5}$ & $9 \%$ & $59 \%$ \\
\hline
\end{tabular}

The data packets are only lost where there is a poor quality of the link established from the consumer to the provider or forwarder vehicle to the consumer (in case of when forwarder vehicles move away from the consumer) or when the selection of the metrics for selecting the best forwarder is not optimized well. The IBFS routing protocol considers the transmission link of the potential relay or forwarder vehicles and focuses on the state of the transmission link to deliver packets successfully. This provides better and reliable routing. As a result, IBFS has a lower ratio of dropped packets when compared with the RUFS as shown in Fig. 19.

In this section, we will consider the experimental results of Interest Satisfaction Ratio in high- 


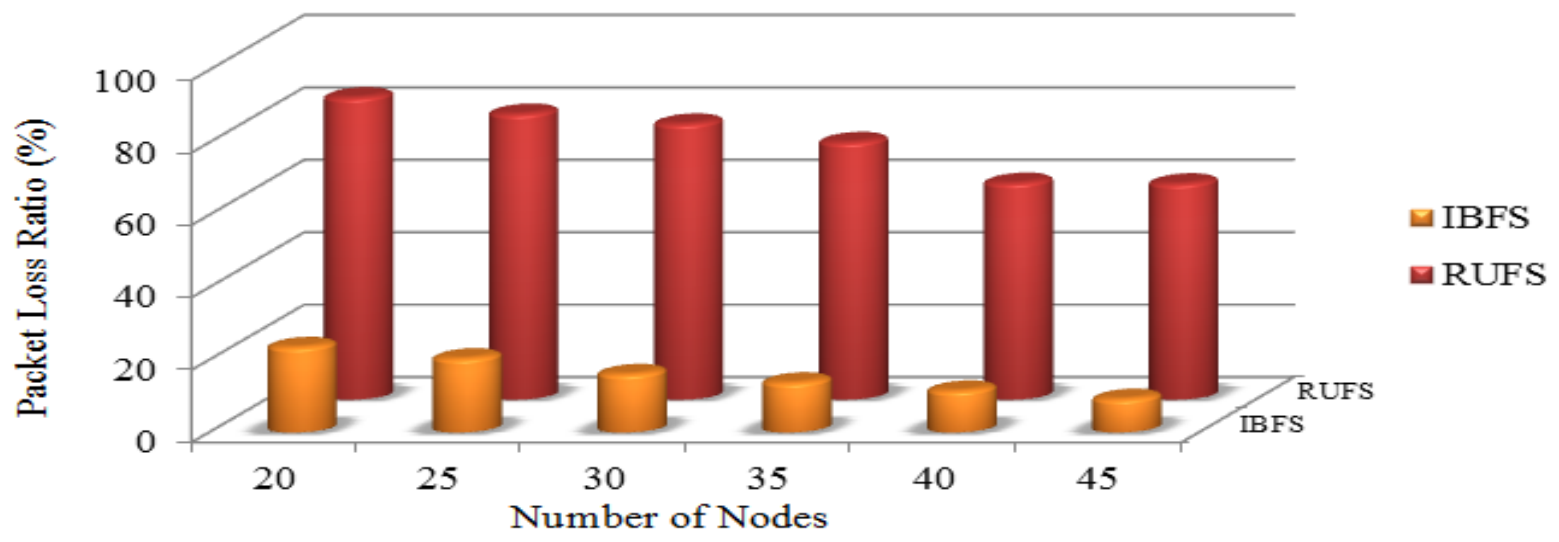

Figure 19: Packet Loss Ratio (PLR) vs Number of Vehicular Nodes in Highway Scenario

\section{way scenario are:}

\subsubsection{Interest Satisfaction Ratio (ISR)}

The Table 6 summarized the total number of interest packets that are fully satisfied at the end of the transmission. In IBFS and RUFS routing protocol, the interest packets are generated randomly at any time. This means that there is no fix time slot in which the interest packet is generated and multiple requests and responses are handled at the same time.

Table 6: Interest Satisfaction Ratio (ISR) Comparison in Highway Scenario

\begin{tabular}{|l|l|l|}
\hline Number of Nodes & IBFS & RUFS \\
\hline $\mathbf{2 0}$ & $84 \%$ & $16 \%$ \\
\hline $\mathbf{2 5}$ & $86 \%$ & $24 \%$ \\
\hline $\mathbf{3 0}$ & $86 \%$ & $25 \%$ \\
\hline $\mathbf{3 5}$ & $91 \%$ & $28 \%$ \\
\hline $\mathbf{4 0}$ & $91 \%$ & $35 \%$ \\
\hline $\mathbf{4 5}$ & $94 \%$ & $40 \%$ \\
\hline
\end{tabular}

In IBFS, the graph of ISR is higher than RUFS because at the end multiple requests of the interest packets are successfully sent at the provider and the provider satisfies more requests of the interest packet as shown in Fig. 20. In this way, IBFS has a high PDR than the RUFS. 


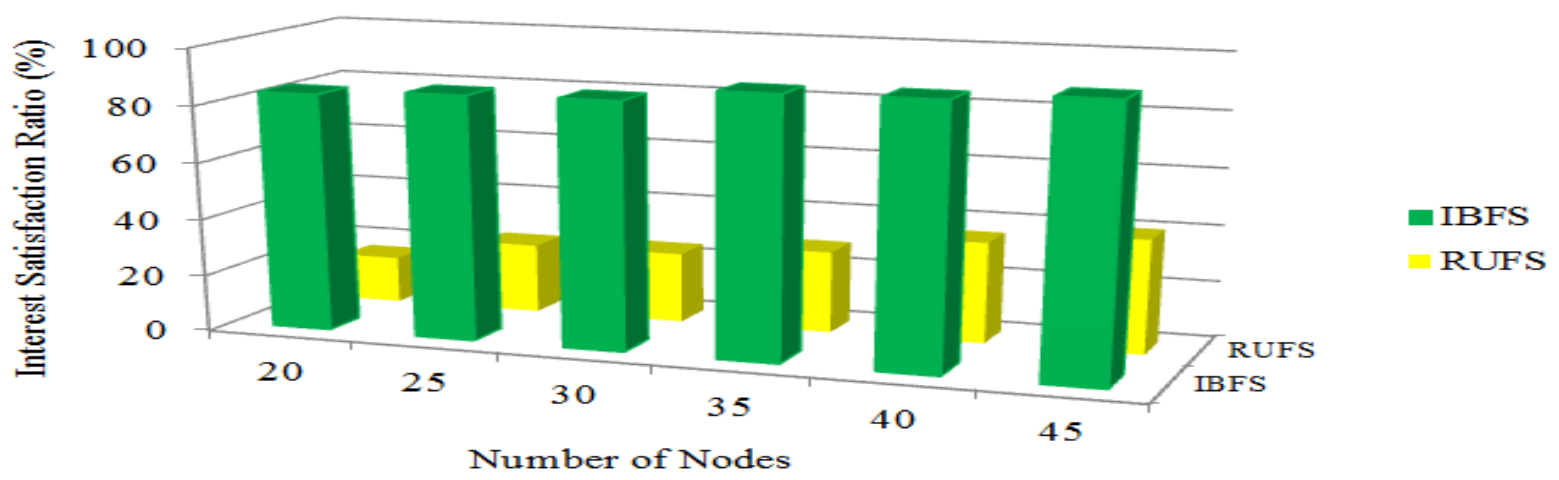

Figure 20: Interest Satisfaction Ratio(ISR) vs Number of Vehicular Nodes in Highway Scenario

\subsubsection{Performance Trade-off}

In the proposed IBFS routing protocol. we achieve better packet delivery ratio as minimum number of interest packets are received at forwarder nodes. Disconnected links are mitigated by introducing the matching trajectory information. The compatible vehicle speed at forwarder nodes lead to the higher lifetime of the packet in the network. IBFS drops least number of packets in a network and the reason is that each vehicle has a high transmission range to find and select a best forwarder. Moreover, AODV a reactive routing scheme is selected to minimum number of interest packets that are generated [58].

The Table 7 presents the average end-to-end delay of IBFS and RUFS forwarding scheme in highway scenario. In this case (i.e., highway), the IBFS has a higher delay than RUFS. This is due to the reason that in the IBFS, the forwarder nodes carry the packet for a certain interval on the basis of matching trajectory and compatible velocity from consumer to provider and provider to forwarder.

Table 7: Average End-to-End Delay Comparison in Highway Scenario

\begin{tabular}{|l|l|l|}
\hline Number of Nodes & IBFS & RUFS \\
\hline $\mathbf{2 0}$ & $0.0032 \%$ & $0.0002 \%$ \\
\hline $\mathbf{2 5}$ & $0.0041 \%$ & $0.0003 \%$ \\
\hline $\mathbf{3 0}$ & $0.0053 \%$ & $0.0005 \%$ \\
\hline $\mathbf{3 5}$ & $0.0052 \%$ & $0.0003 \%$ \\
\hline $\mathbf{4 0}$ & $0.0046 \%$ & $0.0003 \%$ \\
\hline $\mathbf{4 5}$ & $0.0086 \%$ & $0.0002 \%$ \\
\hline
\end{tabular}

Forwarder nodes carry the packet until a reply is received (either content is available or not) from the provider. This consumes time and results in slightly more delay. This is the performance tradeoff of the proposed IBFS as shown in Fig. 21. We analyze the average end-to-end delay and the network load. In proposed IBFS, the average end-to-end delay and the network load is higher than the RUFS. When number of vehicles are increased, the nodes carry the packet until the next forwarder vehicle is not found, the distance between relay vehicles increases and this causes more 
average end-to-end delay.

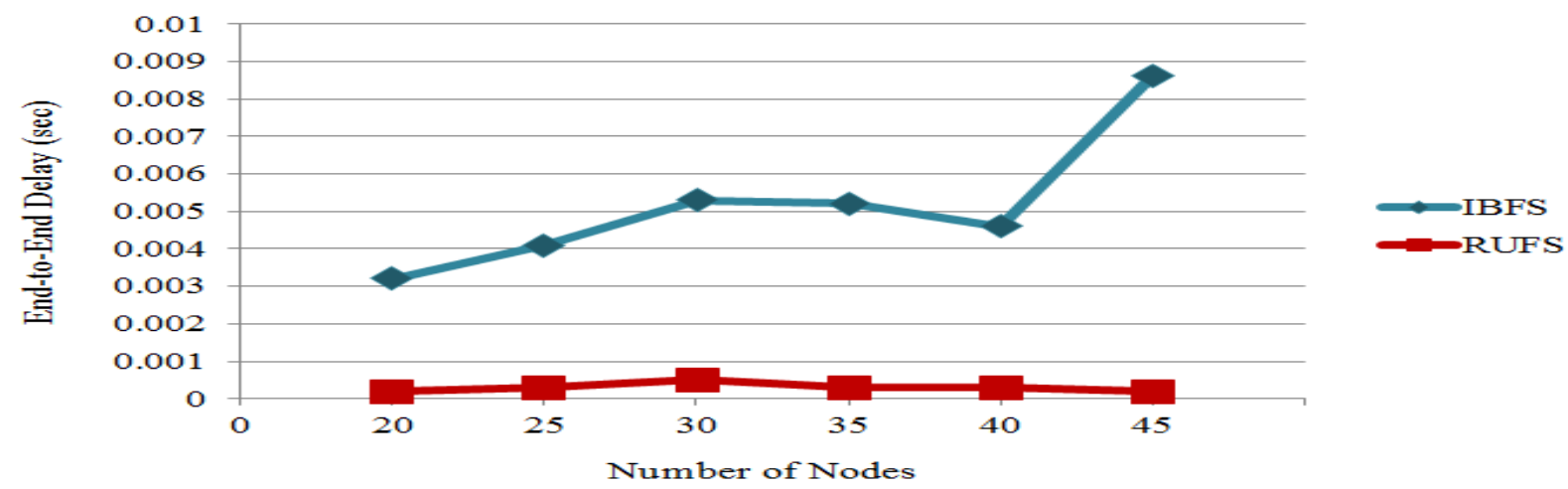

Figure 21: Average End-to-End Delay vs Number of Vehicular Nodes in Highway Scenario

In this section, we will consider the experimental results of urban scenario are:

\subsubsection{Urban Scenario}

The RUFS protocol considers only a highway scenario only. However, the proposed IBFS is evaluated for both highway and urban scenarios. Moreover, for a realistic urban scenario, we obtain the mobility file of the real-time traffic of the Raja Bazaar (Fowara Chowk), Rawalpindi, Pakistan by using Simulation of Urban Mobility (SUMO)[59]. In this scenario 10-45 vehicles are moving with different speeds within a transmission range of 500 meter.

In this section, we will consider the experimental results of Packet Delivery Ratio in urban scenario are:

4.4.3.1 Packet Delivery Ratio (PDR)

In IBFS, the PDR is higher due to higher amount of the packets that are successfully delivered as summarized in Table 8.

Table 8: Packet Delivery Ratio Comparison in Urban Scenario

\begin{tabular}{|l|l|l|}
\hline Number of Nodes & IBFS & RUFS \\
\hline $\mathbf{2 0}$ & $99 \%$ & $95 \%$ \\
\hline $\mathbf{2 5}$ & $99 \%$ & $95 \%$ \\
\hline $\mathbf{3 0}$ & $99 \%$ & $95 \%$ \\
\hline $\mathbf{3 5}$ & $99 \%$ & $96 \%$ \\
\hline $\mathbf{4 0}$ & $99 \%$ & $96 \%$ \\
\hline $\mathbf{4 5}$ & $99 \%$ & $96 \%$ \\
\hline
\end{tabular}

In IBFS, the quality of the stable state of the transmission link in terms of Intersection (i.e., trajectory) and velocity is measured and both the trajectory and the velocity are used as a IBFS parameters to forward data through selected forwarder vehicles. Since, in the highway scenario, more number of packets are delivered and received successfully at provider, same is the case with urban scenario and the PDR is higher than RUFS. Fig. 

are:

\subsubsection{Packet Loss Ratio (PLR)}

We analyze the number of lost packets due to the disconnected link problem. In IBFS, the quality of the transmission link is measured for every link and is used as a performance metric in to select the best forwarder as summarized in Table 9.

Table 9: Packet Loss Ratio Comparison in Urban Scenario

\begin{tabular}{|l|l|l|}
\hline Number of Nodes & IBFS & RUFS \\
\hline $\mathbf{2 0}$ & $0.1 \%$ & $4.1 \%$ \\
\hline $\mathbf{2 5}$ & $0.1 \%$ & $4.1 \%$ \\
\hline $\mathbf{3 0}$ & $0.1 \%$ & $4.3 \%$ \\
\hline $\mathbf{3 5}$ & $0.1 \%$ & $3.6 \%$ \\
\hline $\mathbf{4 0}$ & $0.09 \%$ & $3.48 \%$ \\
\hline $\mathbf{4 5}$ & $0.04 \%$ & $3.37 \%$ \\
\hline
\end{tabular}

This helps in reducing the chances of instable and poor data packet forwarding link.IBFS routing protocol considers the state of the transmission link of potential relay or forwarder vehicles and then selects the best forwarder vehicles. As a result, IBFS has a low number of packet lost ratio when compared to RUFS in an urban scenario. and Fig. 22 presents the performance comparison of IBFS and RUFS.

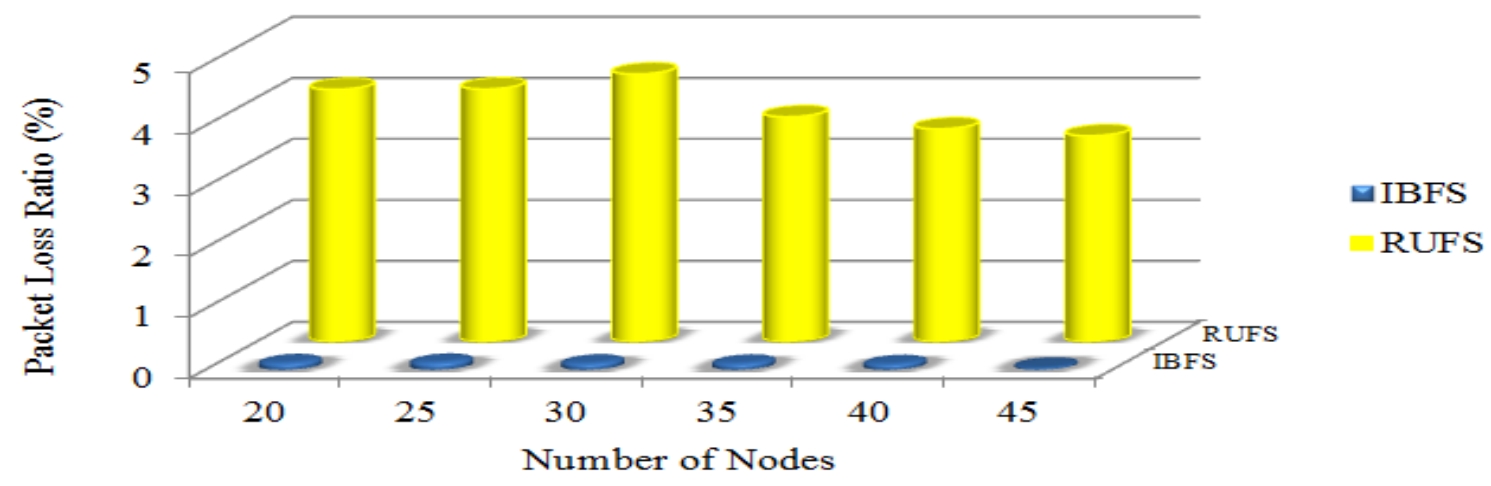

Figure 22: Packet Loss Ratio (PLR) vs Number of Vehicular Nodes in Urban Scenario

In this section, we will consider the experimental results of Interest Satisfaction Ratio in urban scenario are:

\subsubsection{Interest Satisfaction Ratio (ISR)}

In Table 10 IBFS has higher ISR than RUFS because at the end of a transmission, multiple requests of the interest packets are successfully sent at the provider and as a result the consumer receives more responses of the interest packet.

In this way, IBFS has a high PDR than the RUFS. See Fig. 23 which shows the total number of interest packets that are fully satisfied at the end of the transmission. 
Table 10: Interest Satisfaction Ratio (ISR) Comparison in Urban Scenario

\begin{tabular}{|l|l|l|}
\hline Number of Nodes & IBFS & RUFS \\
\hline $\mathbf{2 0}$ & $92 \%$ & $83 \%$ \\
\hline $\mathbf{2 5}$ & $95 \%$ & $85 \%$ \\
\hline $\mathbf{3 0}$ & $98 \%$ & $86 \%$ \\
\hline $\mathbf{3 5}$ & $99 \%$ & $91 \%$ \\
\hline $\mathbf{4 0}$ & $97 \%$ & $91 \%$ \\
\hline $\mathbf{4 5}$ & $98 \%$ & $94 \%$ \\
\hline
\end{tabular}

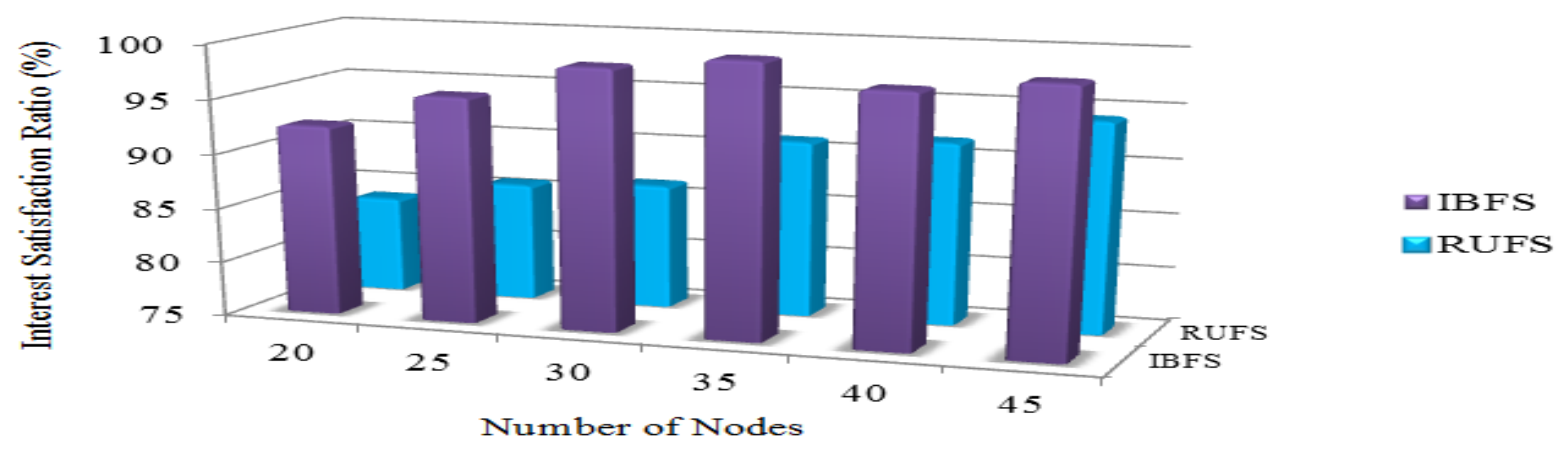

Figure 23: Interest Satisfaction Ratio(ISR) vs Number of Vehicular Nodes in Urban Scenario

In this section, we will consider the experimental results of Average End-to-End Delay in urban scenario are:

4.4.3.4 Average End-to-End Delay

The average delay is calculated and evaluated as summarized in Table 11.

Table 11: Average End-to-End Delay Comparison in Urban Scenario

\begin{tabular}{|l|l|l|}
\hline Number of Nodes & IBFS & RUFS \\
\hline $\mathbf{2 0}$ & $0.7 \%$ & $0.8 \%$ \\
\hline $\mathbf{2 5}$ & $0.7 \%$ & $0.5 \%$ \\
\hline $\mathbf{3 0}$ & $0.7 \%$ & $0.7 \%$ \\
\hline $\mathbf{3 5}$ & $0.8 \%$ & $0.8 \%$ \\
\hline $\mathbf{4 0}$ & $0.8 \%$ & $0.9 \%$ \\
\hline $\mathbf{4 5}$ & $0.9 \%$ & $1.2 \%$ \\
\hline
\end{tabular}

The proposed IBFS has less average delay than RUFS in an urban scenario because the distance from the consumer to the provider and the provider to forwarder is less. The forwarder vehicles take less time to carry the packet in the network as shown in Fig. 24. 


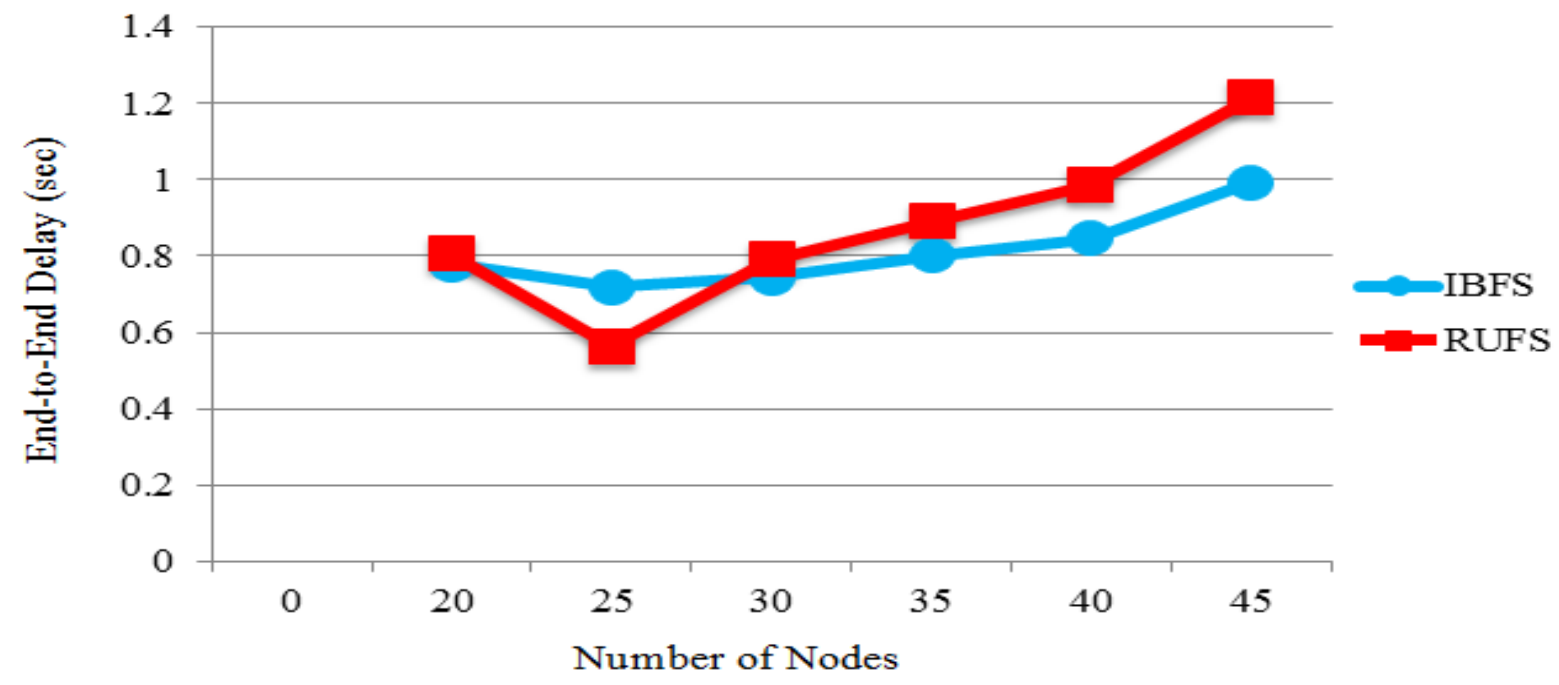

Figure 24: Average End-to-End Delay vs Number of Vehicular Nodes in Urban Scenario

\subsection{Discussion}

In this paper, we have demonstrated big data analytics for sustainability (BDAS) by using VCCN as the example to illustrate. Literature review was discussed and summarized in Section II. The proposed protocol, deployment of different system architecture, supporting algorithms and theoretical development were presented and analyzed in Section III. Results and analysis for different types of BDAS performance evaluation were illustrated and explained thoroughly in Section IV. IBFS is our proposed protocol for VCCN and has better performance evaluation than the commonly-used RUFS protocol. In this way, we conclude that all the step-by-step approach work has made contributions for VCCN with BDAS as the main method for illustrations in scientific steps, algorithms, theories and performance evaluation verified by supporting explanation.

\section{Conclusion}

Our paper has demonstrated a proof-of-concept to develop BDAS, with particular focus for VCCN. The main aim of this paper is to propose a routing protocol for communication in VCCN in order to mitigate the broadcasting storm and disconnected link problems. Our IBFS routing strategy has two phases, first is Intersection Information Sharing phase and the other is Interest/Data packet forwarding phase. For Intersection Information Sharing phase, every vehicle has its own VIT (Vehicle Information Table) and transmission range. When a consumer is interested in a particular content, the consumer vehicle shares its VIT information with its neighboring vehicles. While sharing the VIT information, each vehicle creates NIT (Neighbor Information Table) and stores the information of its neighboring vehicle in its NIT. The stored information helps the vehicle to choose the next best forwarder as rely within its transmission range. In Interest/Data packet forwarding phase, a consumer broadcasts an interest packet within its vicinity and selects a neighbor node as a best forwarder whose intersection information matches and vehicle speed becomes compatible with the consumer vehicle. In this way, we mitigate the disconnected link problem and interest broadcasting storm. Interest packet is forwarded to the provider through the forwarder vehicle. In response, provider generates the data packet and sent back to the consumer through same selected 
forwarder vehicle. Results show that IBFS routing protocol gives good performance in high delivery ratio, less packet loss ratio, high Interest Satisfaction Ratio. However, the proposed IBFS shows a performance trade-off in high average delay in a highway scenario. In urban scenario, it shows good performance with less delay when compared to the existing routing protocol (RUFS). In future, we aim to perform simulation on performance metrics and evaluate the performance parameters that are associated with the IBFS routing protocol in urban as well as highway scenario as compared to the other routing schemes in VCCN. Results and analysis have support and demonstrations for successful BDAS.

\section{References}

[1] A. Feldmann, Internet clean-slate design: what and why?, ACM SIGCOMM Computer Communication Review 37 (3) (2007) 59-64.

[2] D. Clark, The design philosophy of the darpa internet protocols, ACM SIGCOMM Computer Communication Review 18 (4) (1988) 106-114.

[3] V. Jacobson, D. K. Smetters, J. D. Thornton, M. F. Plass, N. H. Briggs, R. L. Braynard, Networking named content, in: Proceedings of the 5th international conference on Emerging networking experiments and technologies, ACM, 2009, pp. 1-12.

[4] F. Zhang, Comparing alternative approaches for mobile content delivery in information-centric networking, in: World of Wireless, Mobile and Multimedia Networks (WoWMoM), 2015 IEEE 16th International Symposium on a, IEEE, 2015, pp. 1-2.

[5] G. Xylomenos, C. N. Ververidis, V. A. Siris, N. Fotiou, C. Tsilopoulos, X. Vasilakos, K. V. Katsaros, G. C. Polyzos, A survey of information-centric networking research, IEEE Communications Surveys \& Tutorials 16 (2) (2014) 1024-1049.

[6] J. Roberts, The clean-slate approach to future internet design: a survey of research initiatives, annals of telecommunications-annales des télécommunications 64 (5-6) (2009) 271-276.

[7] B. Ahlgren, C. Dannewitz, C. Imbrenda, D. Kutscher, B. Ohlman, A survey of information-centric networking, IEEE Communications Magazine 50 (7).

[8] D. Trossen, M. Sarela, K. Sollins, Arguments for an information-centric internetworking architecture, ACM SIGCOMM Computer Communication Review 40 (2) (2010) 26-33.

[9] A. Ghodsi, S. Shenker, T. Koponen, A. Singla, B. Raghavan, J. Wilcox, Information-centric networking: seeing the forest for the trees, in: Proceedings of the 10th ACM Workshop on Hot Topics in Networks, ACM, 2011, p. 1.

[10] D. R. Cheriton, Triad: Translating relaying internetwork architecture integrating active directories: Final report, Tech. rep., DTIC Document (2003).

[11] T. Koponen, M. Chawla, B.-G. Chun, A. Ermolinskiy, K. H. Kim, S. Shenker, I. Stoica, A data-oriented (and beyond) network architecture, in: ACM SIGCOMM Computer Communication Review, Vol. 37, ACM, 2007, pp. 181-192.

[12] N. Fotiou, D. Trossen, G. C. Polyzos, Illustrating a publish-subscribe internet architecture, Telecommunication Systems 51 (4) (2012) 233-245.

[13] C. Dannewitz, D. Kutscher, B. Ohlman, S. Farrell, B. Ahlgren, H. Karl, Network of information (netinf)-an information-centric networking architecture, Computer Communications 36 (7) (2013) 721-735.

[14] P. Murray, et al., Fp7 sail project, D-5.2 (DD. 1) Cloud Network Architecture Description.

[15] L. Zhang, D. Estrin, J. Burke, V. Jacobson, J. D. Thornton, D. K. Smetters, B. Zhang, G. Tsudik, D. Massey, C. Papadopoulos, et al., Named data networking (ndn) project, Relatório Técnico NDN-0001, Xerox Palo Alto Research Center-PARC.

[16] A. Ghodsi, T. Koponen, J. Rajahalme, P. Sarolahti, S. Shenker, Naming in content-oriented architectures, in: Proceedings of the ACM SIGCOMM workshop on Information-centric networking, ACM, 2011, pp. 1-6.

[17] V. Jacobson, M. Mosko, D. Smetters, J. Garcia-Luna-Aceves, Content-centric networking: Whitepaper describing future assurable global networks, Palo Alto Research Center, Inc (2007) 1-9.

[18] S. M. Bilal, C. J. Bernardos, C. Guerrero, Position-based routing in vehicular networks: A survey, Journal of Network and Computer Applications 36 (2) (2013) 685-697.

[19] H. Houda, M. Salah, A survey of trajectory based data forwarding schemes for vehicular ad-hoc networks, in: 
Communication Software and Networks (ICCSN), 2015 IEEE International Conference on, IEEE, 2015, pp. 399-404.

[20] G. Arnould, D. Khadraoui, Z. Habbas, A self-organizing content centric network model for hybrid vehicular adhoc networks, in: Proceedings of the first ACM international symposium on Design and analysis of intelligent vehicular networks and applications, ACM, 2011, pp. 15-22.

[21] B. T. Sharef, R. A. Alsaqour, M. Ismail, Vehicular communication ad hoc routing protocols: A survey, Journal of network and computer applications 40 (2014) 363-396.

[22] B. Paul, M. Ibrahim, M. Bikas, A. Naser, Vanet routing protocols: Pros and cons, arXiv preprint arXiv:1204.1201.

[23] S. H. Bouk, S. H. Ahmed, D. Kim, Vehicular information centric networking: research challenges, in: Proceedings of the Korean Institute of Communications and Information Workshop (KICS'14), 2014.

[24] M. Amadeo, C. Campolo, A. Molinaro, Forwarding strategies in named data wireless ad hoc networks: Design and evaluation, Journal of Network and Computer Applications 50 (2015) 148-158.

[25] Y.-T. Yu, M. Gerla, M. Sanadidi, Scalable vanet content routing using hierarchical bloom filters, Wireless Communications and Mobile Computing 15 (6) (2015) 1001-1014.

[26] S. H. Bouk, S. H. Ahmed, D. Kim, Vehicular content centric network (vccn): a survey and research challenges, in: Proceedings of the 30th Annual ACM Symposium on Applied Computing, ACM, 2015, pp. 695-700.

[27] S. H. Ahmed, S. H. Bouk, D. Kim, Rufs: Robust forwarder selection in vehicular content-centric networks, IEEE Communications Letters 19 (9) (2015) 1616-1619.

[28] S. H. Ahmed, S. H. Bouk, M. A. Yaqub, D. Kim, M. Gerla, Conet: Controlled data packets propagation in vehicular named data networks, in: Consumer Communications \& Networking Conference (CCNC), 2016 13th IEEE Annual, IEEE, 2016, pp. 620-625.

[29] S. H. Ahmed, S. H. Bouk, M. A. Yaqub, D. Kim, H. Song, J. Lloret, Codie: Controlled data and interest evaluation in vehicular named data networks, IEEE Transactions on Vehicular Technology 65 (6) (2016) 39543963.

[30] F. B. Gonçalves, F. M. França, C. L. Amorim, Radnet-ve: An interest-centric mobile ad hoc network for vehicular environments, arXiv preprint arXiv:1604.00589.

[31] M. F. Majeed, S. H. Ahmed, M. N. Dailey, Enabling push-based critical data forwarding in vehicular named data networks, IEEE Communications Letters 21 (4) (2017) 873-876.

[32] D. Saxena, V. Raychoudhury, C. Becker, Implementation and performance evaluation of name-based forwarding schemes in v-ndn, in: Proceedings of the 18th International Conference on Distributed Computing and Networking, ACM, 2017, p. 35.

[33] K. Husain, A. Awang, A receiver-based forwarding scheme to minimize multipath formation in vanet, in: Vehicular Ad-Hoc Networks for Smart Cities, Springer, 2017, pp. 15-26.

[34] D. H. Lee, K. Thar, D. Kim, C. S. Hong, Efficient parallel multi-path interest forwarding for mobile user in ccn, in: Information Networking (ICOIN), 2016 International Conference on, IEEE, 2017, pp. 390-394.

[35] Z. Yan, S. Zeadally, Y.-J. Park, A novel vehicular information network architecture based on named data networking (ndn), IEEE internet of things journal 1 (6) (2014) 525-532.

[36] Z. Zhu, A. Afanasyev, L. Zhang, A new perspective on mobility support, Named-Data Networking Project, Tech. Rep.

[37] D.-h. Kim, J.-h. Kim, Y.-s. Kim, H.-s. Yoon, I. Yeom, Mobility support in content centric networks, in: Proceedings of the second edition of the ICN workshop on Information-centric networking, ACM, 2012, pp. 13-18.

[38] J. Lee, D. Kim, M.-W. Jang, B.-J. Lee, Proxy-based mobility management scheme in mobile content centric networking (ccn) environments, in: Consumer Electronics (ICCE), 2011 IEEE International Conference on, IEEE, 2011, pp. 595-596.

[39] B. Tian, K. M. Hou, J. Li, Trad: Traffic adaptive data dissemination protocol for both urban and highway vanets, in: Advanced Information Networking and Applications (AINA), 2016 IEEE 30th International Conference on, IEEE, 2016, pp. 724-731.

[40] Z. Lin, M. Kuai, X. Hong, Reliable forwarding strategy in vehicular networks using ndn, in: Vehicular Technology Conference (VTC-Fall), 2016 IEEE 84th, IEEE, 2016, pp. 1-5.

[41] L. Wang, R. Wakikawa, R. Kuntz, R. Vuyyuru, L. Zhang, Data naming in vehicle-to-vehicle communications, in: Computer Communications Workshops (INFOCOM WKSHPS), 2012 IEEE Conference on, IEEE, 2012, pp. $328-333$.

[42] L. Wang, A. Afanasyev, R. Kuntz, R. Vuyyuru, R. Wakikawa, L. Zhang, Rapid traffic information dissemination using named data, in: Proceedings of the 1st ACM workshop on emerging name-oriented mobile networking design-architecture, algorithms, and applications, ACM, 2012, pp. 7-12. 
[43] Y.-B. Ko, N. H. Vaidya, Geocasting in mobile ad hoc networks: Location-based multicast algorithms, in: Mobile Computing Systems and Applications, 1999. Proceedings. WMCSA'99. Second IEEE Workshop on, IEEE, 1999, pp. 101-110.

[44] S. H. Ahmed, M. Yaqub, S. Bouk, D. Kim, Towards content-centric traffic ticketing in vanets: An application perspective, in: Ubiquitous and Future Networks (ICUFN), 2015 Seventh International Conference on, IEEE, 2015, pp. 237-239.

[45] H. Wu, R. Fujimoto, R. Guensler, M. Hunter, Mddv: a mobility-centric data dissemination algorithm for vehicular networks, in: Proceedings of the 1st ACM international workshop on Vehicular ad hoc networks, ACM, 2004, pp. 47-56.

[46] G. Deng, X. Xie, L. Shi, R. Li, Hybrid information forwarding in vanets through named data networking, in: Personal, Indoor, and Mobile Radio Communications (PIMRC), 2015 IEEE 26th Annual International Symposium on, IEEE, 2015, pp. 1940-1944.

[47] C. Lochert, H. Hartenstein, J. Tian, H. Fussler, D. Hermann, M. Mauve, A routing strategy for vehicular ad hoc networks in city environments, in: Intelligent Vehicles Symposium, 2003. Proceedings. IEEE, IEEE, 2003, pp. 156-161.

[48] M. Amadeo, C. Campolo, A. Molinaro, Content-centric networking: is that a solution for upcoming vehicular networks?, in: Proceedings of the ninth ACM international workshop on Vehicular inter-networking, systems, and applications, ACM, 2012, pp. 99-102.

[49] M. Amadeo, C. Campolo, A. Molinaro, Crown: Content-centric networking in vehicular ad hoc networks, IEEE Communications Letters 16 (9) (2012) 1380-1383.

[50] G. Grassi, D. Pesavento, G. Pau, L. Zhang, S. Fdida, Navigo: Interest forwarding by geolocations in vehicular named data networking, in: World of Wireless, Mobile and Multimedia Networks (WoWMoM), 2015 IEEE 16th International Symposium on a, IEEE, 2015, pp. 1-10.

[51] F. F. Qureshi, R. Iqbal, M. N. Asghar, Energy efficient wireless communication technique based on cognitive radio for internet of things, Journal of Network and Computer Applications 89 (2017) 14-25.

[52] C. Bian, T. Zhao, X. Li, W. Yan, Boosting named data networking for efficient packet forwarding in urban vanet scenarios, in: Local and Metropolitan Area Networks (LANMAN), 2015 IEEE International Workshop on, IEEE, 2015, pp. 1-6.

[53] W. Chen, R. K. Guha, T. J. Kwon, J. Lee, Y.-Y. Hsu, A survey and challenges in routing and data dissemination in vehicular ad hoc networks, Wireless Communications and Mobile Computing 11 (7) (2011) 787-795.

[54] T. Nadeem, P. Shankar, L. Iftode, A comparative study of data dissemination models for vanets, in: Mobile and Ubiquitous Systems-Workshops, 2006. 3rd Annual International Conference on, IEEE, 2006, pp. 1-10.

[55] D. L. Olson, Comparison of weights in topsis models, Mathematical and Computer Modelling 40 (7-8) (2004) $721-727$.

[56] Y. Wang, O.-C. Yue, A tutorial of 802.11 implementation in ns-2, arXiv preprint arXiv:1202.3576.

[57] Y. J. Li, An overview of the dsrc/wave technology, in: International Conference on Heterogeneous Networking for Quality, Reliability, Security and Robustness, Springer, 2010, pp. 544-558.

[58] S. Sethi, S. K. Udgata, Optimized and reliable aodv for manet, International Journal of Computer Application $3(10)$.

[59] C. Raj, U. Upadhayaya, T. Makwana, P. Mahida, Simulation of vanet using ns-3 and sumo, International Journal $4(4)$. 